\title{
ASYMPTOTICS OF SOME NONLINEAR EIGENVALUE PROBLEMS FOR A MEMS CAPACITOR: PART I: FOLD POINT ASYMPTOTICS*
}

\author{
A. E. LINDSAY ${ }^{\dagger}$ AND M. J. WARD ${ }^{\dagger \ddagger}$
}

\begin{abstract}
Several nonlinear eigenvalue problems modeling the steady-state deflection of an elastic membrane associated with a MEMS capacitor under a constant applied voltage are analyzed using formal asymptotic methods. The nonlinear eigenvalue problems under consideration represent various regular and singular perturbations of the basic membrane nonlinear eigenvalue problem $\Delta u=\lambda /(1+u)^{2}$ in $\Omega$ with $u=0$ on $\partial \Omega$, where $\Omega$ is a bounded two-dimensional domain. The following three perturbations of this basic problem are considered; the addition of a bending energy term of the form $-\delta \Delta^{2} u$; the effect of a fringing-field where $\lambda$ is replaced by $\lambda\left(1+\delta|\nabla u|^{2}\right)$, and the effect of including a small inner undeflected disk of radius $\delta$. For each of the perturbed problems an asymptotic expansion of the fold point location $\lambda_{c}$ at the end of minimal solution branch in the limit $\delta \rightarrow 0$ is constructed. This calculation determines the pull-in voltage threshold, which is critical for the design of a MEMS device. In addition, with regards to solution multiplicity, it is shown numerically that the effect of each of the perturbations is to destroy the well-known infinite fold point behavior associated with the bifurcation diagram of the basic membrane problem in the unit
\end{abstract} disk.

Key words. Nonlinear eigenvalue problem, Fold point, Matched asymptotic expansions, Boundary Layer, Shooting, Psuedo-arclength continuation.

AMS subject classifications. 34E05, 35B25, 35B32

1. Introduction. Micro-Electromechanical Systems (MEMS) combine electronics with micro-size mechanical devices to design various types of microscopic machinery (cf. [18]). A key component of many MEMS systems is the simple MEMS capacitor shown in Fig. 1. The upper part of this device consists of a thin deformable elastic plate that is held clamped along its boundary, and which lies above a fixed ground plate. When a voltage $V$ is applied to the upper plate, the upper plate can exhibit a significant deflection towards the lower ground plate.

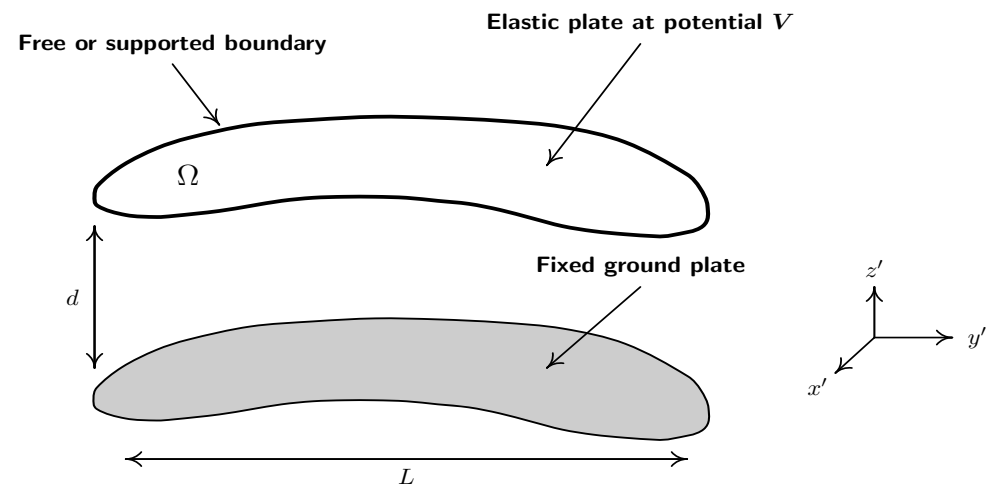

FIG. 1. Schematic plot of the MEMS capacitor with a deformable elastic upper surface that deflects towards the fixed lower surface under an applied voltage

\footnotetext{
${ }^{*}$ Received August 10, 2008; accepted for publication October 21, 2008.

${ }^{\dagger}$ Department of Mathematics, University of British Columbia, Vancouver, British Columbia, V6T 1Z2, Canada.

${ }^{\ddagger}$ ward@math.ubc.ca
} 
By including the effect of a bending energy it was shown in [18] (see also [16] and the references therein) that the dimensionless steady state deflection $u(x)$ of the upper plate satisfies the fourth-order nonlinear eigenvalue problem

$$
-\delta \Delta^{2} u+\Delta u=\frac{\lambda}{(1+u)^{2}}, \quad x \in \Omega ; \quad u=\partial_{n} u=0 \quad x \in \partial \Omega .
$$

Here, the positive constant $\delta$ represents the relative effects of tension and rigidity on the deflecting plate, and $\lambda \geq 0$ represents the ratio of electric forces to elastic forces in the system and is directly proportional to the square of the voltage $V$ applied to the upper plate. The boundary conditions in (1.1) assume that the upper plate is in an undeflected and clamped state along the rim of the plate. The model (1.1) was derived in [18] from a narrow-gap asymptotic analysis.

A special limiting case of (1.1) is when $\delta=0$, so that the upper surface is modeled by a membrane rather than a plate. Omitting the requirement that $\partial_{n} u=0$ on $\partial \Omega$, (1.1) reduces to the MEMS membrane problem

$$
\Delta u=\frac{\lambda}{(1+u)^{2}}, \quad x \in \Omega ; \quad u=0 \quad x \in \partial \Omega
$$

This simple nonlinear eigenvalue problem has been studied using formal asymptotic analysis in [19] and [9] for the unit slab and the unit disk. For the unit disk, one of the key qualitative features for (1.2) is that the bifurcation diagram $|u|_{\infty}$ versus $\lambda$ for radially symmetric solutions of (1.2) has an infinite number of fold points with $\lambda \rightarrow 4 / 9$ as $|u|_{\infty} \rightarrow 1$ (cf. [19]). Analytical bounds for the pull-in voltage instability threshold, representing the fold point location $\lambda_{c}$ at the end of the minimal solution branch for (1.2), have been derived (cf. [19], [8], [9]). A generalization of (1.2) that has received considerable interest from a mathematical viewpoint is the following problem with a variable permittivity profile $|x|^{\alpha}$ in an $N$-dimensional domain $\Omega$ :

$$
\Delta u=\frac{\lambda|x|^{\alpha}}{(1+u)^{2}}, \quad x \in \Omega ; \quad u=0 \quad x \in \partial \Omega .
$$

There are now many rigorous results for (1.2) and (1.3) in the unit ball in dimension $N$ and in more general domains $\Omega$. In particular, upper and lower bounds for $\lambda_{c}$ have been derived for (1.2) and for (1.3) for the range of parameters $\alpha$ and $N$ where solution multiplicity occurs (cf. [8]). In [12] it has been proved that there are an infinite number of fold points for (1.2) in a certain class of symmetric domains. Many other rigorous results for solution multiplicity for (1.3) under various ranges of $\alpha$ and $N$ have been obtained in [8], [6], and [11].

In contrast to (1.2) and (1.3), there are only a few rigorous results available for (1.1) and its fourth-order variants. Under Navier boundary conditions $u=\Delta u=0$ on $\partial \Omega$, the existence of a maximal solution for (1.1) was proved in [16] and its uniqueness established in [14]. Under Navier boundary conditions and in the three-dimensional unit ball it was proved in [13] that $-\Delta^{2} u=\lambda /(1+u)^{2}$ has infinitely many fold points for the bifurcation branch corresponding to radially symmetric solutions. In [5], the regularity of the minimal solution branch together with bounds for the "pull-in voltage" for the corresponding clamped problem $-\Delta^{2} u=\lambda /(1+u)^{2}$ with $u=\partial_{n} u=$ 0 on $|x|=1$ in the $N$-dimensional unit ball are established for $N \leq 8$. Some related rigorous results are given in [2].

In [21] the effect on the pull-in voltage of the electric field at the edge of a diskshaped membrane of finite extent was analyzed by deriving a uniform expansion for 
the electric field that includes edge effects. It was shown in [21] that such edge effects induce a global perturbation of the basic nonlinear eigenvalue problem (1.2). From equation (34) of [21] the perturbed problem for $\delta \ll 1$ is

$$
\Delta u=\frac{\lambda}{(1+u)^{2}}\left(1+\delta|\nabla u|^{2}\right), \quad x \in \Omega ; \quad u=0, \quad x \in \partial \Omega .
$$

Here $\delta=(d / L)^{2}$ is an aspect ratio, where $d$ is the gap width between the upper and lower surfaces, and $L$ is the lengthscale of each of the surfaces (see Fig. 1). For the unit disk, (1.4) was studied numerically in $\S 5$ of [21], where it was shown that the effect of the fringing-field is to reduce the pull-in voltage. In addition, it was shown qualitatively in [21] that the effect of the fringing-field is to destroy the infinite fold point structure of the basic membrane problem (1.2) in the unit disk.

Another simple modification of the membrane problem (1.2) in the unit disk is to pin the rim of a concentric inner disk in the undeflected state (cf. [20], [7]). The perturbed problem for $\delta \ll 1$ in the concentric circular domain $0<\delta<|x|<1$ is formulated as

$$
\Delta u=\frac{\lambda}{(1+u)^{2}}, \quad 0<\delta<r<1 ; \quad u=0 \text { on }|x|=1 \text { and }|x|=\delta .
$$

This change in the topology of the membrane from inserting a small inner disk has a two-fold effect on the solution. Firstly, it increases the pull-in voltage rather significantly. Secondly, it allows for the existence of non-radially symmetric solutions that bifurcate off of the radially symmetric solution branch (cf. [20], [7]).

For $\delta \ll 1$, the problems (1.1), (1.4), and (1.5), can all be viewed as perturbations of the basic and well-studied membrane problem (1.2). The primary goal of this paper is to determine how the pull-in voltage threshold for (1.2) gets perturbed for $\delta \ll 1$ under these three perturbations of the basic model. A rather precise determination of the pull-in voltage threshold is required for the actual design of a MEMS capacitor since, typically, the operating range of the device is chosen rather close to the pull-in instability threshold (cf. [18], [21]). Therefore, in mathematical terms, our primary goal is to calculate asymptotic expansions for the fold point location $\lambda_{c}$ at the end of the minimal solution branch for (1.1), (1.4), and (1.5), in the limit $\delta \rightarrow 0$. In the companion paper [17], a quantitative asymptotic theory describing the destruction of the infinite fold points for (1.2) in the unit disk when (1.2) is perturbed for $0<\delta \ll 1$ to either the biharmonic problem (1.1), the fringing-field problem (1.4), or the annulus problems (1.5), is developed.

For the biharmonic problem (1.1) asymptotic expansions for the fold point location, denoted by $\lambda_{c}$, at the end of the minimal solution branch are derived in the limiting parameter ranges $\delta \ll 1$ and $\delta \gg 1$ for an arbitrary domain $\Omega$ with smooth boundary. To treat the $\delta \ll 1$ limit of (1.1), singular perturbation techniques are used to resolve the boundary layer near the boundary $\partial \Omega$ of $\Omega$, which is induced by the term $\delta \Delta^{2} u$ in (1.1). This analysis yields effective boundary conditions for the corresponding outer solution, which is defined away from an $\mathcal{O}\left(\delta^{1 / 2}\right)$ neighborhood of $\partial \Omega$. Then, appropriate solvability conditions are imposed to determine analytical formulae for the coefficients in the asymptotic expansion of $\lambda_{c}$ for $\delta \ll 1$. These coefficients are evaluated numerically for the unit slab and the unit disk. In contrast, the analysis of (1.1) for the limiting case $\delta \gg 1$ consists of a regular perturbation expansion off of the solution to the pure Biharmonic nonlinear eigenvalue problem $-\Delta^{2} u=\tilde{\lambda} /(1+u)^{2}$, 
with $\tilde{\lambda} \equiv \lambda / \delta$. In this way it is shown for the unit disk and the unit slab that

$$
\begin{aligned}
\lambda_{c} & \sim 70.095 \delta+1.729+\cdots, \quad \delta \gg 1 ; \\
\lambda_{c} & \sim 1.400+5.600 \delta^{1 / 2}+25.451 \delta+\cdots, \quad \delta \ll 1 ; \quad(\text { Unit Slab) }, \\
\lambda_{c} & \sim 15.412 \delta+1.001+\cdots, \quad \delta \gg 1 ; \\
\lambda_{c} & =0.789+1.578 \delta^{1 / 2}+6.261 \delta+\cdots, \quad \delta \ll 1 ; \quad \text { (Unit Disk) } .
\end{aligned}
$$

It is shown below in Fig. 8, Fig. 6(b), and Fig. 7(b), that (1.6) compares very well with full numerical results for $\lambda_{c}$ computed from (1.1). In particular, rather remarkably, Fig. 8 below shows that the asymptotic result for $\lambda_{c}$ in (1.6) derived for the $\delta \gg 1$ limit can give a reliable estimate of $\lambda_{c}$ even for $\delta \approx 0.03$. The asymptotic results in (1.6) for $\delta \ll 1$ accurately predict $\lambda_{c}$ for $0<\delta<0.03$ (see Fig. 6(b) and Fig. 7(b)). Therefore, for the unit slab and the unit disk we conclude that (1.6) gives a rather accurate estimate of $\lambda_{c}$ for (1.1) for essentially the entire range $0<\delta<\infty$, and hence (1.6) can give a good prediction of the pull-in voltage for (1.1). This conclusion is one of the main results of this paper.

For $\delta \ll 1$ it is shown that through an analysis of the fold point location $\lambda_{c}$ for (1.4) that the effect of a fringing-field is to reduce the pull-in voltage by an amount of $\mathcal{O}(\delta)$ for $\delta \ll 1$. For the unit disk, it is calculated through a solvability condition that $\lambda_{c} \sim 0.789-0.160 \delta$ for $\delta \ll 1$. This asymptotic result is shown to compare very favorably with full numerical results computed from (1.4).

For $\delta \ll 1$ a singular perturbation analysis of the fold point location $\lambda_{c}$ for the annulus problem (1.5) shows that $\lambda_{c} \sim 0.789+\mathcal{O}(-1 / \log \delta)$. The coefficient of this logarithmic term, which is evaluated numerically, is shown to be positive. Therefore, the effect of the inner disk of radius $\delta$ is to perturb the pull-in voltage for the membrane problem (1.2) rather significantly even when $\delta \ll 1$. Some related nonlinear eigenvalue problems with small holes were treated in [24] and [23].

The outline of this paper is as follows. In $\S 2$ some numerical approaches for the computation of solutions of (1.1), (1.2), (1.4), and (1.5), are discussed and results are given for the corresponding bifurcation diagrams $|u|_{\infty}$ versus $\lambda$. For the unit slab and unit disk, a simple upper bound for the fold point location $\lambda_{c}$ at the end of the minimal solution branch for (1.1) is derived and calculated numerically. In $\S 3$ and $\S 4$ asymptotic expansions for the fold point location $\lambda_{c}$ for (1.1) are derived in the limit $\delta \rightarrow 0$ for the unit slab and for a multi-dimensional domain, respectively. In $\S 5$ an asymptotic expansion for this fold point location for (1.1) in the limit $\delta \gg 1$ is constructed. Finally, in $\S 6$ an asymptotic expansion for the fold point location for the minimal solution branch of the fringing-field problem (1.4) and the annulus (1.5) are obtained. Brief conclusions are given in $\S 6$.

2. Numerical Solution of Some Nonlinear Eigenvalue Problems. In this section, an outline of the numerical methods used to compute the bifurcation diagrams associated with the nonlinear eigenvalue problems (1.1), (1.2), (1.4), and (1.5), is provided. The results of these computations provide motivation for the asymptotic analysis in $\S 3-6$ and are useful for validating our asymptotic results.

For the membrane problem (1.2), the use of scale invariance as a computational technique to compute bifurcation diagrams was explored in [19]. By introducing the new variables $t$ and $w$ by

$$
u(r)=-1+\alpha w(y), \quad y=t r
$$


it was shown in [19] that the bifurcation diagram for (1.2) can be parameterized as

$$
|u(0)|=1-\frac{1}{w(\eta)}, \quad \lambda=\frac{\eta^{2}}{w^{3}(\eta)}, \quad \eta>0,
$$

where $w(\eta)$ is the solution of the initial value problem

$$
w^{\prime \prime}+\frac{1}{\eta} w^{\prime}=\frac{1}{w^{2}}, \quad \eta>0 ; \quad w(0)=1, \quad w^{\prime}(0)=0 .
$$

By solving (2.2) numerically, the bifurcation diagram, as shown in Fig. 2, is obtained. For this problem, it was shown in [19] that there are an infinite number of fold points that have the limiting behavior $|u(0)| \rightarrow 1^{-}$as $\lambda \rightarrow 4 / 9$. A similar scale invariance method can also be used for computing solutions to the generalized membrane problem (1.3).

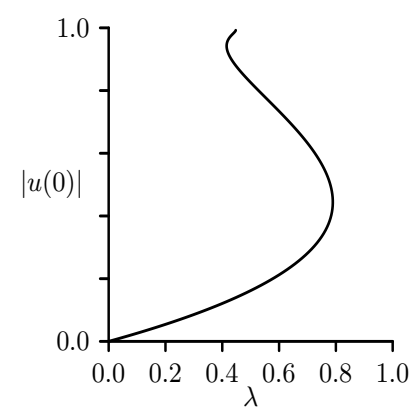

(a) Unit Disk: Membrane Problem

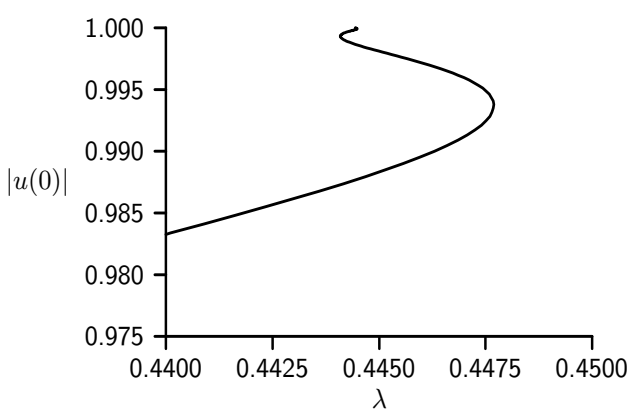

(b) Unit Disk (Zoomed): Membrane Problem

FIG. 2. Numerical solutions for $|u(0)|$ versus $\lambda$ computed from (1.2) for the unit disk $\Omega=$ $\{|x| \leq 1\}$ in two-dimensions. The magnified figure on the right shows the beginning of the infinite set of fold points.

Next, the scale invariance method is extended to compute solutions to the fourthorder problem (1.1) for the two-dimensional unit disk. By introducing new variables $v$ and $y$ by

$$
u=-1+\alpha v(y), \quad y=\operatorname{Tr},
$$

equation (1.1) becomes

$$
-\delta \alpha T^{4} \Delta_{y}^{2} v+\alpha T^{2} \Delta_{y} v=\frac{\lambda}{\alpha^{2} v^{2}} .
$$

The conditions $u^{\prime}(0)=u^{\prime \prime \prime}(0)=0$ imply that $v^{\prime}(0)=v^{\prime \prime \prime}(0)=0$. The free parameter $v(0)$ is chosen as $v(0)=1$ so that $u(0)=-1+\alpha$. Enforcing the boundary condition $u(1)=0$ requires that $\alpha=1 / v(T)$, while $u^{\prime}(1)=0$ is satisfied if $v^{\prime}(T)=0$. Finally, by letting $\lambda=\alpha^{3} T^{4}$, a parametric form of the bifurcation diagram is given by

$$
|u(0)|=1-\frac{1}{v(T)}, \quad \lambda=\frac{T^{4}}{v^{3}(T)},
$$

where $v(y)$ is the solution of

$-\delta \Delta_{y}^{2} v+\frac{1}{T^{2}} \Delta_{y} v=\frac{1}{v^{2}}, \quad 0 \leq y \leq T ; \quad v(0)=1, \quad v^{\prime}(0)=0, \quad v^{\prime \prime \prime}(0)=0, v^{\prime}(T)=0$. 
There are two options for solving (2.3). The first option, representing a shooting approach, consists of solving (2.3) as an initial value problem and choosing the value of $v^{\prime \prime}(0)$ so that $v^{\prime}(T)=0$. The second option is to solve (2.3) directly as a boundary value problem. For this approach it is convenient to rescale the interval to $[0,1]$ by making the transformation $y \rightarrow T y$, resulting in

$-\delta \Delta_{y}^{2} v+\Delta_{y} v=\frac{T^{4}}{v^{2}}, \quad 0 \leq y \leq 1 ; \quad v(0)=1, \quad v^{\prime}(0)=0, \quad v^{\prime \prime \prime}(0)=0, \quad v^{\prime}(1)=0$.

In these variables, the bifurcation diagram for (1.1) is then parameterized in terms of $T$ by

$$
|u(0)|=1-\frac{1}{v(1)}, \quad \lambda=\frac{T^{4}}{v^{3}(1)},
$$

where $v(y)$ is the solution to (2.4). A similar approach is used to compute the bifurcation diagram of (1.1) in a slab.

It is remarked that the solution of the membrane problem (1.2) using the scale invariance method requires that the initial value problem (2.2) be solved exactly once. In contrast, for the fourth-order problem (1.1) the solution of either (2.3) or (2.4) must be computed for each point on the bifurcation branch. However, notice that in contrast to solving (1.1) directly using a two-parameter shooting method, the scale invariance method leading to (2.3) involves only a one-parameter shooting.

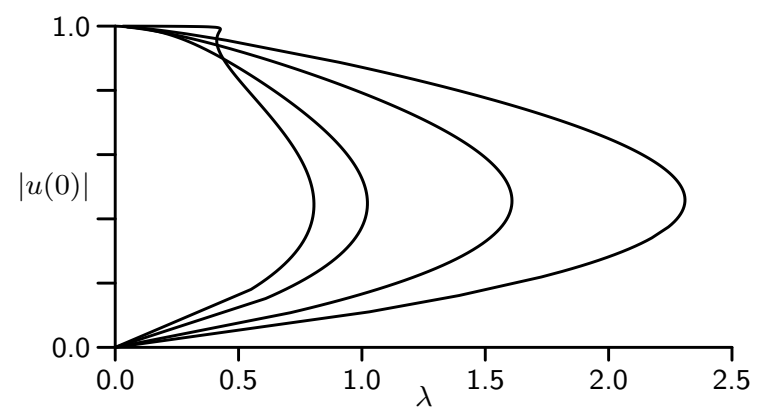

(a) Unit Disk

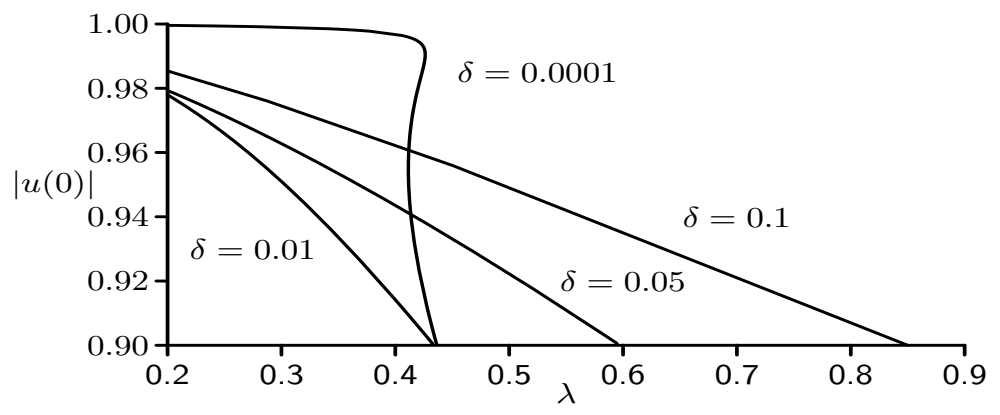

(b) Unit Disk (Zoomed)

FIG. 3. Numerical solutions of (1.1) for the unit disk $\Omega=\{|x| \leq 1\}$ for several values of $\delta$. From left to right the solution branches correspond to $\delta=0.0001,0.01,0.05,0.1$. The figure on the right is a magnification of a portion of the left figure.

In Fig. 3 the numerically computed bifurcation diagram of $|u(0)|$ versus $\lambda$ is 
plotted for $\Omega=\{|x| \leq 1\}$ and for various values of $\delta>0$ with $\delta$ small. These numerical results indicate that the presence of the biharmonic term in (1.1) with small nonzero coefficient $\delta$ destroys the infinite fold point behavior associated with the membrane problem (1.2) shown previously in Fig. 2. Furthermore, numerical results suggest the existence of some critical value $\delta_{c}<<1$, such that for $\delta>\delta_{c}$ equation (1.1) exhibits either zero, one, or two, solutions, with the resulting bifurcation diagram having only one fold point at the end of the minimal solution branch. In $\S 4$ an asymptotic expansion of the fold point at the end of the minimal solution branch for (1.1) in powers of $\delta^{1 / 2}$ for $\delta \ll 1$ is developed. In $\S 3$ the corresponding problem in the unit slab is considered. In $\S 5$ an asymptotic expansion of the fold point for (1.1) when $\delta \gg 1$ for both the unit disk and the unit slab is constructed.

To numerically compute the bifurcation diagram associated with the fringing-field problem (1.4) in the unit disk the numerically observed fact that the solution can be uniquely characterized by the value of $u(0)$ is exploited. By assigning a range of values to $u(0)$ in the interval $(-1,0),(1.4)$ is solved as an initial value problem and the value of $\lambda$ is uniquely determined by the zero of $g(\lambda)=u(1 ; \lambda)$. A Newton iteration scheme is implemented on $g(\lambda)$ with initial guess $u(0)=\lambda=0$. This method was found to be effective provided the stepsize in $u(0)$ is sufficiently small.

In order to numerically treat the annulus problem (1.5) it is advantageous to rescale the domain to $[0,1]$ with the change of variables $\rho=(r-\delta) /(1-\delta)$. Then (1.5) transforms to

$$
\frac{d^{2} u}{d \rho^{2}}+\frac{(1-\delta)}{\delta+(1-\delta) \rho} \frac{d u}{d \rho}=\frac{\lambda(1-\delta)^{2}}{(1+u)^{2}}, \quad 0<\rho<1 ; \quad u(0)=u(1)=0 .
$$

In a way similar to the numerical approach for the fringing-fields problem (1.4), solutions to (2.6) are computed at predetermined values of $u^{\prime}(0)<0$ so that $(2.6)$ becomes an initial value problem. The value of $\lambda$ is then fixed by the unique zero of $g(\lambda)=u(1 ; \lambda)$, which is computed using Newton's method.

The numerically computed bifurcation diagrams for the fringing-fields problem (1.4) and the annulus problem (1.5) are plotted in Fig. 4(a) and Fig. 4(b), respectively, for various values of $\delta$. It is again observed that, for $\delta$ small, that the effect of the perturbation is to destroy the infinite fold point behavior associated with the membrane problem (1.2). In $\S 6$ asymptotic results for the location of the fold point at the end of the minimal solution branch are given for (1.4) and (1.5) when $\delta \ll 1$.

A straightforward approach to compute solutions to (1.1), (1.4), and (1.5), is to solve the underlying ODE boundary value problems by using a standard boundary value solver such as COLSYS [1]. This approach works well provided that the bifurcation diagram can be parameterized in terms of the coordinate on the vertical axis of the bifurcation diagram, such as $u(0)$. Then, the BVP solver can be formulated to solve for $u(x)$ and $\lambda$.

Next, a more general approach to the numerical solution of the bifurcation branch of (1.1) is described, which also applies to a multi-dimensional domain $\Omega$. For the unit square $\Omega=[0,1] \times[0,1],(1.1)$ is not amenable to the scale invariance technique and a more general approach based on pseudo-arclength continuation (cf. [15]) is required. This method take a direct approach to compute solutions of the general problem

$$
f: \mathbb{R}^{n} \times \mathbb{R} \rightarrow \mathbb{R}^{n}, \quad f(u, \lambda)=0 .
$$

Starting with an initial solution $\left(u_{0}, \lambda_{0}\right)$, the method seeks to determine a sequence of points $\left(u_{j}, \lambda_{j}\right)$ which satisfy $(2.7)$ to within some specified tolerance. 


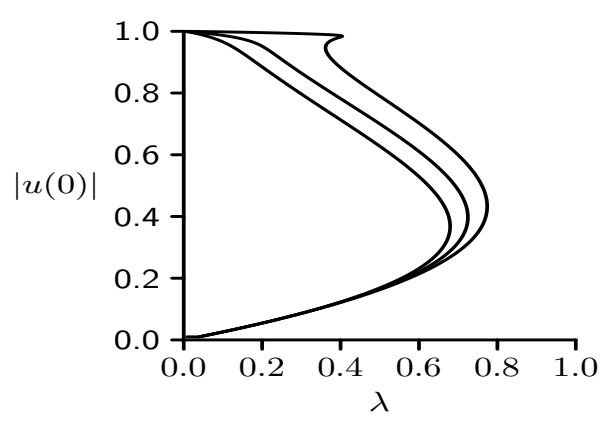

(a) Unit Disk: Fringing Field

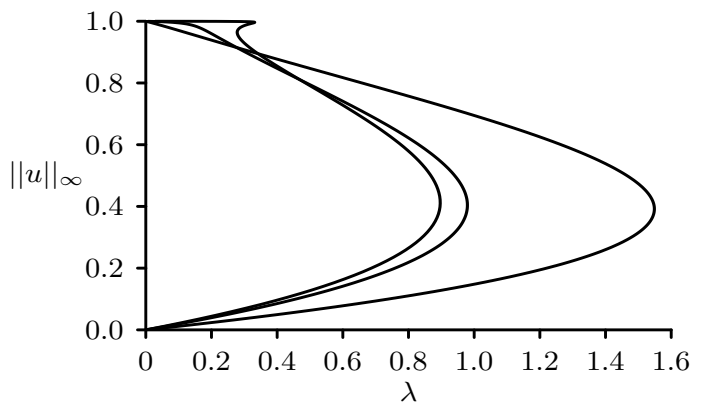

(b) Annulus

FiG. 4. Left figure: Numerical solutions for $|u(0)|$ versus $\lambda$ computed from the fringing-field problem (1.4) for the unit disk $\Omega=\{|x| \leq 1\}$ with, from left to right, $\delta=1,0.5,0.1$. Right figure: Numerical solutions for $|u|_{\infty}$ versus $\lambda$ computed from (1.5) in the annulus $\delta<r<1$ with, from left to right, $\delta=0.00001,0.001,0.1$.

The following is a brief outline of this method based on [15]. In order to compute solutions around fold points at which the system has a singular jacobian and the bifurcation curve has a vertical tangent, the method introduces a parameterization of the curve $n(u(s), \lambda(s), d s)=0$ in terms of an arclength parameter $s$ and seeks new points on the solution branch at predetermined values of the steplength $d s$. To choose $n(u(s), \lambda(s), d s)=0$, consider some accepted point $\left(u_{j}, \lambda_{j}\right)$ and its tangent vector to the curve at that point $\left(\dot{u}_{j}, \dot{\lambda}_{j}\right)$, where an overdot represents differentiation with respect to arclength $s$. Now, define

$$
n(u(s), \lambda(s), d s)=\dot{u}_{j}^{T} \cdot\left(u-u_{j}\right)+\dot{\lambda}_{j}\left(\lambda-\lambda_{j}\right)-d s,
$$

as the hyperplane whose normal vector is $\left(\dot{u}_{j}, \dot{\lambda}_{j}\right)$ and whose perpendicular distance from $\left(u_{j}, \lambda_{j}\right)$ is $d s$. The intersection of this hyperplane with the bifurcation curve will be non-zero provided the curvature of the branch and $d s$ are not too large. With this specification of $n$, the pseudo-arclength continuation method seeks a solution to the augmented system

$$
f(u(s), \lambda(s))=0, \quad n(u(s), \lambda(s), s)=0,
$$

which is non-singular at simple fold points (cf. [15]). Applying Newton's method with initial guess $\left(u_{j}, \lambda_{j}\right)$ to the solution of $(2.9)$ results in the following iteration scheme: 


$$
\begin{gathered}
\left(\begin{array}{cc}
f_{u}\left(u^{(k)}, \lambda^{(k)}\right) & f_{\lambda}\left(u^{(k)}, \lambda^{(k)}\right) \\
\dot{u}_{j}^{T} & \dot{\lambda}_{j}
\end{array}\right)\left(\begin{array}{c}
\Delta u \\
\Delta \lambda
\end{array}\right)=-\left(\begin{array}{c}
f\left(u^{(k)}, \lambda^{(k)}\right) \\
n\left(u^{(k)}, \lambda^{(k)}\right)
\end{array}\right) \\
u^{(k+1)}=u^{(k)}+\Delta u \\
\lambda^{(k+1)}=\lambda^{(k)}+\Delta \lambda
\end{gathered}
$$

By differentiating (2.7) with respect to $\lambda$ and solving the resulting linear system $f_{u} u_{\lambda}+f_{\lambda}=0$, the tangent vector $(\dot{u}, \dot{\lambda})$ is specified as

$$
\dot{u}=a u_{\lambda}, \quad \dot{\lambda}=a, \quad a=\frac{ \pm 1}{\sqrt{1+\left\|u_{\lambda}\right\|_{2}^{2}}} .
$$

The sign of $a$ is chosen to preserve the direction in which the branch is traversed.

To compute solutions of (1.1) by the pseudo-arclength method in the unit square $\Omega=[0,1] \times[0,1]$, the partial derivatives are approximated by central finite difference quotients, which results in a large system of nonlinear equations. In Fig. 5(b) the numerically computed bifurcation diagram for (1.1) in the unit square is plotted for several values of $\delta$. The computations were done with a uniform mesh-spacing of $h=1 / 100$ in the $x$ and $y$ directions. The bifurcation diagram is similar to that of the unit disk shown in Fig. 3(a). In Fig. 5(a) the corresponding numerical bifurcation diagram for (1.1) in the one-dimensional unit slab is plotted.

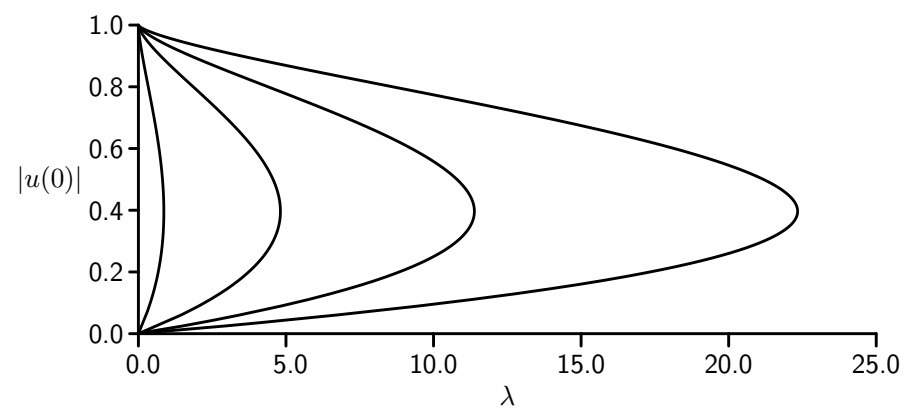

(a) Unit Slab

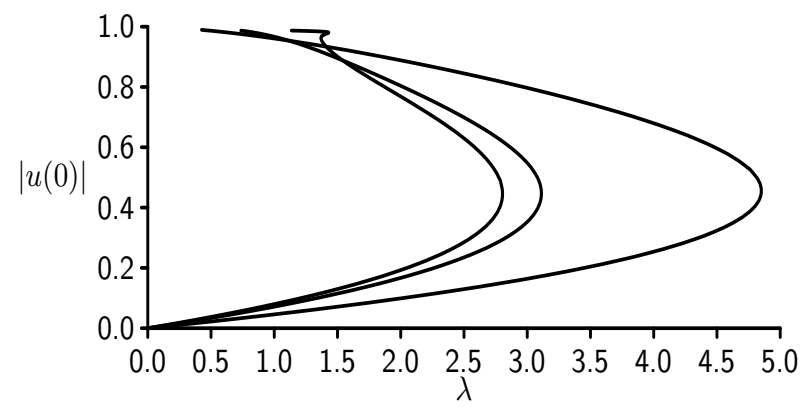

(b) Unit Square

FIG. 5. Numerical solutions of (1.1) for the slab $0<x<1$ (left figure) and the unit square for several values of $\delta$. From left to right the solution branches correspond to $\delta=0.1,1.0,2.5,5.0$ (left figure) and $\delta=0.0001,0.001,0.01$ (right figure). 
A quantitative asymptotic theory describing the destruction of the infinite fold points for (1.2) when (1.2) is perturbed for $0<\delta \ll 1$ to either the biharmonic problem (1.1), the fringing-field problem (1.4), or the annulus problems (1.5), is given in the companion paper [17]. This is done by constructing the limiting form of the bifurcation diagram when $|u|_{\infty}=1-\varepsilon$, where $\varepsilon \rightarrow 0^{+}$. In [17] asymptotic results for the limiting behavior $\lambda \rightarrow 0$ and $\varepsilon \rightarrow 0$ of the maximal solution branch are also presented for these perturbed problems.

2.1. Simple upper bounds for $\lambda_{c}$. In the case where $\Omega$ represents either the unit slab or the unit disk, a simple upper bound for the fold point location at the end of the minimal solution branch, $\lambda_{c}$, is obtained for (1.1). The existence of this bound demonstrates that $\lambda_{c}$ is finite and provides a rather good estimate of its value. The bound is established in terms of the principal eigenvalue of the differential operator appearing on the left hand side of (1.1). Therefore the associated eigenvalue problem requires the determination of a function $\phi$ and a scalar $\mu$ such that

$$
-\delta \Delta^{2} \phi+\Delta \phi=-\mu \phi, \quad x \in \Omega ; \quad \phi=\partial_{n} \phi=0, \quad x \in \partial \Omega .
$$

When $\Omega$ is either the unit slab or the unit disk the positivity of the first eigenfunction $\phi_{0}$ is verified numerically from the explicit formulae for $\phi_{0}$ given below in (2.16) and (2.17). Owing to the lack of a maximum principle, the positivity of the first eigenfunction for (2.11) is not guaranteed for more general domains. In particular, for domains such as squares or rectangles or annuli, the principle eigenfunction of the limiting problem $\delta \rightarrow \infty$ in (2.11) is known to change sign (cf. [3], [4]). For a survey of such results see [22]. Therefore, the following discussion is limited to either the unit slab or the unit disk.

To derive an upper bound for $\lambda_{c}$, the approach in [19] needs to be modified only slightly. We assume that $u$ exists and use Green's second identity on $u$ and the principal eigenfunction $\phi_{0}$ and eigenvalue $\mu_{0}$ of (2.11) to obtain

$$
\begin{aligned}
0 & =\delta \int_{\Omega}\left(-\phi_{0} \Delta^{2} u+u \Delta^{2} \phi_{0}\right) d x \\
& =\int_{\Omega} \phi_{0}\left(\frac{\lambda}{(1+u)^{2}}+\mu_{0} u\right) d x-\int_{\Omega}\left(\phi_{0} \Delta u-u \Delta \phi_{0}\right) d x .
\end{aligned}
$$

The second integral on the right-hand side of (2.12) vanishes identically, and so a necessary condition for a solution to (1.1) is that

$$
\int_{\Omega} \phi_{0}\left(\frac{\lambda}{(1+u)^{2}}+\mu_{0} u\right) d x=0
$$

Since $\phi_{0}>0$, then there is no solution to $(2.13)$ when

$$
\frac{\lambda}{(1+u)^{2}}+\mu_{0} u>0, \quad \forall u>-1 .
$$

By considering the point at which the inequality (2.14) ceases to hold, it is clear that there is no solution to (1.1) when $\lambda>4 \mu_{0} / 27$ and therefore

$$
\lambda_{c} \leq \bar{\lambda} \equiv \frac{4 \mu_{0}}{27},
$$

where $\mu_{0}$ is the first eigenvalue of (2.11). 


\begin{tabular}{|c|c|c|c|c|}
\hline & \multicolumn{2}{|c|}{ Slab } & \multicolumn{2}{c|}{ Unit Disc } \\
\hline \hline$\delta$ & $\lambda$ & $\lambda_{c}$ & $\lambda$ & $\lambda_{c}$ \\
0.25 & 20.3576 & 19.249 & 4.886 & 4.395 \\
0.5 & 38.900 & 36.774 & 8.754 & 7.871 \\
1.0 & 75.979 & 71.823 & 16.486 & 14.826 \\
2.0 & 150.137 & 141.918 & 31.948 & 28.704 \\
\hline
\end{tabular}

TABLE 1

Upper bounds, $\bar{\lambda}_{c}$, for the fold point location given in (2.15) compared with the numerically computed fold point location $\lambda_{c}$ of (1.1).

For the unit slab $0<x<1$, a simple calculation shows that the eigenfunctions of (2.11) are given up to a scalar multiple by

$$
\phi=\cosh \left(\xi_{1} x\right)-\cos \left(\xi_{2} x\right)-\left(\frac{\xi_{2} \sinh \left(\xi_{1} x\right)-\xi_{1} \sin \left(\xi_{2} x\right)}{\xi_{2} \sinh \xi_{1}-\xi_{1} \sin \xi_{2}}\right)\left(\cosh \xi_{1}-\cos \xi_{2}\right)
$$

Here $\xi_{1}>0$ and $\xi_{2}>0$ are defined in terms of $\mu$ by

$$
\xi_{1}=\sqrt{\frac{1+\sqrt{1+4 \mu \delta}}{2 \delta}}, \quad \xi_{2}=\sqrt{\frac{-1+\sqrt{1+4 \mu \delta}}{2 \delta}},
$$

where the eigenvalues $\mu$ are the roots of the transcendental equation

$$
2 \xi_{1}+\left(\frac{\xi_{1}^{2}-\xi_{2}^{2}}{\xi_{2}}\right) \sin \xi_{2} \sinh \xi_{1}-2 \xi_{1} \cosh \xi_{1} \cos \xi_{2}=0
$$

Similarly, for the unit disk $0<r<1$, the eigenfunctions are given up up to a scalar multiple by

$$
\phi=J_{0}\left(\xi_{2} r\right)-\frac{J_{0}\left(\xi_{2}\right)}{I_{0}\left(\xi_{1}\right)} I_{0}\left(\xi_{1} r\right),
$$

where $J_{0}$ and $I_{0}$ are the Bessel and modified Bessel functions of the first kind of order zero, respectively. The eigenvalues $\mu$ are the roots of the transcendental equation

$$
\xi_{1} I_{1}\left(\xi_{1}\right)+\xi_{2} \frac{I_{0}\left(\xi_{1}\right)}{J_{0}\left(\xi_{2}\right)} J_{1}\left(\xi_{2}\right)=0,
$$

where $J_{1}(\rho)=-J_{0}^{\prime}(\rho)$ and $I_{1}(\rho)=I_{0}^{\prime}(\rho)$.

The first root of (2.16c) and (2.17b) corresponding to the principle eigenvalue, $\mu_{0}$, of (2.11) is readily computed using Newton's method as a function of $\delta>0$. Then, the corresponding principal eigenfunction $\phi_{0}$ from either $(2.16 \mathrm{a})$ or $(2.17 \mathrm{a})$ can be readily verified numerically to have one sign on $\Omega$. Then, in terms of $\mu_{0},(2.15)$ gives an explicit upper bound for $\lambda_{c}$. These bounds for $\lambda_{c}$ together with the full numerical results for $\lambda_{c}$ are compared in Table 1 . From this table it is observed that the upper bound provides is actually relatively close to the true location for the fold point.

3. Biharmonic Nonlinear Eigenvalue Problem: Slab Geometry. In this section an asymptotic expansion for the fold point at the end of the minimal solution branch for (1.1) is developed in a slab domain. This fold point determines the onset 
of the pull-in instability, and hence its determination is important in the actual design of a MEMS device.

In a slab domain (1.1) becomes

$$
-\delta u^{\prime \prime \prime \prime}+u^{\prime \prime}=\frac{\lambda}{(1+u)^{2}}, \quad 0<x<1 ; \quad u(0)=u(1)=u^{\prime}(0)=u^{\prime}(1)=0 .
$$

In the limit $\delta \ll 1,(3.1)$ is a singular perturbation problem for which the solution $u$ has boundary layers near both endpoints $x=0$ and $x=1$. The width of these boundary layers is found below to be $\mathcal{O}\left(\delta^{1 / 2}\right)$, which then induces an asymptotic expansion for the fold point location in powers of $\mathcal{O}\left(\delta^{1 / 2}\right)$. Therefore, in the outer region defined away from $\mathcal{O}\left(\delta^{1 / 2}\right)$ neighborhoods of both endpoints, $u$ and $\lambda$ are expanded as

$$
u=u_{0}+\delta^{1 / 2} u_{1}+\delta u_{2}+\cdots, \quad \lambda=\lambda_{0}+\delta^{1 / 2} \lambda_{1}+\delta \lambda_{2}+\cdots .
$$

Upon substituting (3.2) into (3.1), and collecting powers of $\delta^{1 / 2}$, the following sequence of problems is obtained

$$
\begin{aligned}
u_{0}^{\prime \prime} & =\frac{\lambda_{0}}{\left(1+u_{0}\right)^{2}}, \quad 0<x<1, \\
\mathcal{L} u_{1} & =\frac{\lambda_{1}}{\left(1+u_{0}\right)^{2}}, \quad 0<x<1, \\
\mathcal{L} u_{2} & =\frac{\lambda_{2}}{\left(1+u_{0}\right)^{2}}-\frac{2 \lambda_{1} u_{1}}{\left(1+u_{0}\right)^{3}}+\frac{3 \lambda_{0} u_{1}^{2}}{\left(1+u_{0}\right)^{4}}+u_{0}^{\prime \prime \prime \prime}, \quad 0<x<1 .
\end{aligned}
$$

Here $\mathcal{L}$ is the linear operator defined by

$$
\mathcal{L} \phi \equiv \phi^{\prime \prime}+\frac{2 \lambda_{0}}{\left(1+u_{0}\right)^{3}} \phi .
$$

Next, appropriate boundary conditions for $u_{0}, u_{1}$ and $u_{2}$ as $x \rightarrow 0$ and $x \rightarrow 1$ are determined. These conditions are obtained by matching the outer solution to boundary layer solutions defined in the vicinity of $x=0$ and $x=1$.

In the boundary layer region near $x=1$, the following inner variables $y$ and $v(y)$ are introduced together with the inner expansion for $v$;

$$
y=\delta^{-1 / 2}(x-1), \quad u=\delta^{1 / 2} v, \quad v=v_{0}+\delta^{1 / 2} v_{1}+\delta v_{2}+\cdots .
$$

Upon substitution of (3.5) and (3.2) for $\lambda$ into (3.1), powers of $\delta^{1 / 2}$ are collected to obtain on $-\infty<y<0$ that

$$
\begin{aligned}
& -v_{0}^{\prime \prime \prime \prime}+v_{0}^{\prime \prime}=0, \quad v_{0}(0)=v_{0}^{\prime}(0)=0, \\
& -v_{1}^{\prime \prime \prime \prime}+v_{1}^{\prime \prime}=\lambda_{0}, \quad v_{1}(0)=v_{1}^{\prime}(0)=0, \\
& -v_{2}^{\prime \prime \prime \prime}+v_{2}^{\prime \prime}=\lambda_{1}-2 \lambda_{0} v_{0}, \quad v_{2}(0)=v_{2}^{\prime}(0)=0 .
\end{aligned}
$$

The solution to (3.6) with no exponential growth as $y \rightarrow-\infty$ is given in terms of unknown constants $c_{0}, c_{1}$, and $c_{2}$, by

$$
\begin{aligned}
& v_{0}=c_{0}\left(-1-y+e^{y}\right), \\
& v_{1}=c_{1}\left(-1-y+e^{y}\right)+\lambda_{0} y^{2} / 2, \\
& v_{2}=c_{2}\left(-1-y+e^{y}\right)+\lambda_{1} y^{2} / 2+c_{0} \lambda_{0} y\left(-1+y+e^{y}+y^{2} / 3\right) .
\end{aligned}
$$


The matching condition to the outer solution is obtained by letting $y \rightarrow-\infty$ and substituting $v_{0}, v_{1}$, and $v_{2}$ into (3.5) for $u$, and then writing the resulting expression in terms of outer variables. In this way, the following matching condition as $x \rightarrow 1$ is established:

$$
\begin{aligned}
u \sim-c_{0}(x-1) & +\mathcal{O}\left((x-1)^{2}\right)+\delta^{1 / 2}\left[-c_{0}-c_{1}(x-1)+\mathcal{O}\left((x-1)^{2}\right)\right] \\
& +\delta\left[-c_{1}-\left(c_{0} \lambda_{0}+c_{2}\right)(x-1)+\mathcal{O}\left((x-1)^{2}\right)\right]+\cdots
\end{aligned}
$$

This matching condition not only gives appropriate boundary conditions to the outer problems for $u_{0}, u_{1}$, and $u_{2}$, defined in (3.3), but it also determines the unknown constants $c_{0}, c_{1}$, and $c_{2}$ in the inner solutions (3.7) in a recursive way. In particular, the $\mathcal{O}\left(\delta^{0}\right)$ term in (3.8) yields that $u_{0}=0$ at $x=1$ and that $c_{0}$ is then given by $c_{0}=-u_{0}^{\prime}(1)$. The remaining $\mathcal{O}\left(\delta^{0}\right)$ terms in (3.8) then match identically as seen by using the solution $u_{0}$ to (3.3a). In a similar way, boundary conditions for $u_{1}$ and $u_{2}$ and formulae for the constants $c_{1}$ and $c_{2}$ are established. A similar analysis can be performed for the boundary layer region at the other endpoint $x=0$. This analysis is identical to that near $x=1$ since $u_{0}, u_{1}$ and $u_{2}$ are symmetric about the mid-line $x=1 / 2$.

In this way, the following boundary conditions for (3.3) are obtained:

$$
u_{0}(0)=u_{0}(1)=0, \quad u_{1}(0)=u_{1}(1)=u_{0}^{\prime}(1), \quad u_{2}(0)=u_{2}(1)=u_{1}^{\prime}(1) .
$$

The constants $c_{0}, c_{1}$, and $c_{2}$, in (3.7) that are associated with the boundary layer solution near $x=1$ are given by

$$
c_{0}=-u_{0}^{\prime}(1), \quad c_{1}=-u_{1}^{\prime}(1), \quad c_{2}=-u_{2}^{\prime}(1)+\lambda_{0} u_{0}^{\prime}(1),
$$

which then determines the boundary layer solution in (3.7) explicitly.

Therefore, (3.3) for $u_{0}, u_{1}$, and $u_{2}$, must be solved subject to the boundary conditions as given in (3.9). With the introduction of $\alpha=u_{0}(1 / 2)$, a parameterization of the minimal solution branch for $u_{0}$ and $\lambda_{0}$ is established and the dependence $u_{j}=$ $u_{j}(x, \alpha)$ for $j=0,1,2$ follows. It is readily verified that the solution to (3.3b) is given by (see Lemma 3.2 below)

$$
u_{1}=\frac{\lambda_{1}}{3 \lambda_{0}}\left(1+u_{0}\right)
$$

where $\lambda_{1}$ is found by satisfying $u_{1}(1)=u_{0}^{\prime}(1)$. Therefore, for $\delta \ll 1$, the following explicit two-term expansions for both the outer solution and for the global bifurcation curve $\lambda(\alpha)$ is developed:

$$
\begin{aligned}
u & \sim u_{0}(x ; \alpha)+\delta^{1 / 2} u_{0}^{\prime}(1, \alpha)\left[1+u_{0}(x, \alpha)\right]+\mathcal{O}(\delta), \\
\lambda & \sim \lambda_{0}(\alpha)+3 \lambda_{0}(\alpha) u_{0}^{\prime}(1, \alpha) \delta^{1 / 2}+\mathcal{O}(\delta) .
\end{aligned}
$$

It is noted that this "global" perturbation result for $\lambda$ is not uniformly valid in the limit $\alpha \rightarrow-1$ corresponding to $\lambda_{0} \rightarrow 0$. In this limit, the term $\left(1+u_{0}\right)^{-2}$ is nearly singular at $x=1 / 2$, and a different asymptotic analysis is required (see $\S 5$ of [17]).

A higher-order local analysis of the bifurcation diagram near the fold point on the minimal solution branch is now constructed. This minimal solution branch for $u_{0}$ is well-known to have a fold point at $\alpha=\alpha_{0} \approx-0.389$ at which $\lambda_{c} \equiv \lambda_{0}\left(\alpha_{0}\right) \approx 1.400$. This point determines the pull-in voltage for the unperturbed problem. To determine 
the location of the fold point for the perturbed problem, expand $\alpha(\delta)=\alpha_{0}+\delta^{1 / 2} \alpha_{1}+$ $\delta \alpha_{2}$, where $\alpha_{j}$ is determined by the condition that $d \lambda / d \alpha=0$ is independent of $\delta$. Defining $\lambda_{c}(\delta)=\lambda(\alpha(\delta), \delta)$, the expansion of the fold point for $(3.1)$ when $\delta \ll 1$ is determined to be

$$
\lambda_{c}=\lambda_{0 c}+\delta^{1 / 2} \lambda_{1}\left(\alpha_{0}\right)+\delta\left[\lambda_{2}\left(\alpha_{0}\right)-\frac{\lambda_{1 \alpha}^{2}\left(\alpha_{0}\right)}{2 \lambda_{0 \alpha \alpha}\left(\alpha_{0}\right)}\right]+\mathcal{O}\left(\delta^{3 / 2}\right) .
$$

Here the subscript indicates derivatives in $\alpha$.

Therefore, to determine a three-term expansion for the fold point as in (3.13), the quantities $\lambda_{1}, \lambda_{2}, \lambda_{1 \alpha}$ and $\lambda_{0 \alpha \alpha}$ must be calculated at the unperturbed fold point location $\alpha_{0}$ from the solution to (3.3) with boundary conditions (3.9). To do so, the problems for $u_{0}$ and $u_{1}$ in (3.3) are first differentiated with respect to $\alpha$ to obtain on $0<x<1$ that

$$
\begin{aligned}
\mathcal{L} u_{0 \alpha} & =\frac{\lambda_{0 \alpha}}{\left(1+u_{0}\right)^{2}}, \\
\mathcal{L} u_{0 \alpha \alpha} & =\frac{\lambda_{0 \alpha \alpha}}{\left(1+u_{0}\right)^{2}}-\frac{4 \lambda_{0 \alpha} u_{0 \alpha}}{\left(1+u_{0}\right)^{3}}+\frac{6 \lambda_{0} u_{0 \alpha}^{2}}{\left(1+u_{0}\right)^{4}} \\
\mathcal{L} u_{1 \alpha} & =\frac{\lambda_{1 \alpha}}{\left(1+u_{0}\right)^{2}}-\frac{2 \lambda_{1} u_{0 \alpha}}{\left(1+u_{0}\right)^{3}}-\frac{2 \lambda_{0 \alpha} u_{1}}{\left(1+u_{0}\right)^{3}}+\frac{6 \lambda_{0} u_{1} u_{0 \alpha}}{\left(1+u_{0}\right)^{4}} .
\end{aligned}
$$

Here $\mathcal{L}$ is the linear operator defined in (3.4). At the unperturbed fold location $\alpha=\alpha_{0}$, where $\lambda_{0 \alpha}=0$, the function $u_{0 \alpha}$ is a nontrivial solution satisfying $\mathcal{L} u_{0 \alpha}=0$. Therefore, $\lambda_{1}\left(\alpha_{0}\right), \lambda_{2}\left(\alpha_{0}\right), \lambda_{0 \alpha \alpha}\left(\alpha_{0}\right)$, and $\lambda_{1 \alpha}\left(\alpha_{0}\right)$, can be calculated by applying a Fredholm solvability condition to each of $(3.3 \mathrm{~b}),(3.3 \mathrm{c}),(3.14 \mathrm{~b})$, and (3.14c), respectively. Upon applying Lagrange's identity to (3.14a) and (3.3b) at $\alpha=\alpha_{0}$, the following equality is established:

$$
\int_{0}^{1} u_{0 \alpha} \mathcal{L} u_{1} d x=\int_{0}^{1} u_{1} \mathcal{L} u_{0 \alpha} d x=-u_{1}(1) u_{0 \alpha}^{\prime}(1)+u_{1}(0) u_{0 \alpha}^{\prime}(0) .
$$

Therefore, since $u_{1}(1)=u_{1}(0)=u_{0}^{\prime}(1)$ from (3.9), and $u_{0 \alpha}^{\prime}(1)=-u_{0 \alpha}^{\prime}(0)$, it follows at $\alpha=\alpha_{0}$ that

$$
\lambda_{1} I=-2 u_{0}^{\prime}(1) u_{0 \alpha}^{\prime}(1), \quad I \equiv \int_{0}^{1} \frac{u_{0 \alpha}}{\left(1+u_{0}\right)^{2}} d x .
$$

The integral $I$ can be evaluated more readily using the following lemma:

Lemma 3.1. At $\alpha=\alpha_{0}$, the following identity holds:

$$
I \equiv \int_{0}^{1} \frac{u_{0 \alpha}}{\left(1+u_{0}\right)^{2}} d x=-\frac{2}{3 \lambda_{0}} u_{0 \alpha}^{\prime}(1) .
$$

To prove this lemma, $(3.14 \mathrm{a})$ is first multiplied by $\left(1+u_{0}\right)$ and then integrated over $0<x<1$. Setting $\alpha=\alpha_{0}$, integrating by parts twice, and then using $u_{0}^{\prime \prime}=$ $\lambda_{0} /\left(1+u_{0}\right)^{2}$, results in the following sequence of equalities:

$$
\begin{aligned}
I=-\frac{1}{2 \lambda_{0}} \int_{0}^{1}\left(1+u_{0}\right) u_{0 \alpha}^{\prime \prime} d x & =-\frac{1}{2 \lambda_{0}}\left[2 u_{0 \alpha}^{\prime}(1)+\int_{0}^{1} u_{0 \alpha} u_{0}^{\prime \prime} d x\right] \\
& =-\frac{u_{0 \alpha}^{\prime}(1)}{\lambda_{0}}-\frac{1}{2} \int_{0}^{1} \frac{u_{0 \alpha}}{\left(1+u_{0}\right)^{2}} d x
\end{aligned}
$$


This last expression gives $I=-u_{0 \alpha}^{\prime}(1) / \lambda_{0}-I / 2$ which is rearranged to yield (3.16), and completes the proof of Lemma 3.1.

Next, (3.16) is substituted into (3.15) and evaluated at $\alpha=\alpha_{0}$ to reveal that

$$
\lambda_{1}=3 \lambda_{0} u_{0}^{\prime}(1) .
$$

This result is consistent with the global perturbation result (3.12) when it is evaluated at $\alpha=\alpha_{0}$.

The values of $\lambda_{0 \alpha \alpha}, \lambda_{1 \alpha}$, and $\lambda_{0 \alpha \alpha}$, at $\alpha=\alpha_{0}$ can be evaluated by imposing similar solvability conditions with respect to $u_{0 \alpha}$. From (3.14a) and (3.14b), and by using (3.16) for $I$, it is readily shown at $\alpha=\alpha_{0}$ that

$$
\lambda_{0 \alpha \alpha}=\frac{9 \lambda_{0}^{2}}{u_{0 \alpha}^{\prime}(1)} \int_{0}^{1} \frac{u_{0 \alpha}^{3}}{\left(1+u_{0}\right)^{4}} d x .
$$

Next, from (3.14a) and (3.14c), we calculate at $\alpha=\alpha_{0}$ that

$$
\int_{0}^{1} u_{0 \alpha} \mathcal{L} u_{1 \alpha} d x=-\left.u_{1 \alpha} u_{0 \alpha}^{\prime}\right|_{0} ^{1}=-2 u_{1 \alpha}(1) u_{0 \alpha}^{\prime}(1) .
$$

Upon using (3.14c) for $\mathcal{L} u_{1 \alpha}$ and $u_{1 \alpha}(1)=u_{0 \alpha}^{\prime}(1)$ from (3.9), the expression above becomes

$$
\lambda_{1 \alpha} I=-\int_{0}^{1} u_{0 \alpha}\left(\frac{6 \lambda_{0} u_{1} u_{0 \alpha}}{\left(1+u_{0}\right)^{4}}-\frac{2 \lambda_{1} u_{0 \alpha}}{\left(1+u_{0}\right)^{3}}\right) d x-2\left[u_{0 \alpha}^{\prime}(1)\right]^{2}
$$

In a similar way, $\lambda_{2}$ is evaluated at $\alpha=\alpha_{0}$ by application of Lagrange's identity to (3.14a) and (3.3c) to obtain

$$
\lambda_{2} I=-\int_{0}^{1} u_{0 \alpha}\left[\frac{3 \lambda_{0} u_{1}^{2}}{\left(1+u_{0}\right)^{4}}-\frac{2 \lambda_{1} u_{1}}{\left(1+u_{0}\right)^{3}}+u_{0}^{\prime \prime \prime \prime}\right] d x-2 u_{0 \alpha}^{\prime}(1) u_{1}^{\prime}(1) .
$$

The formulae above for $\lambda_{1 \alpha}$ and $\lambda_{2}$ at $\alpha=\alpha_{0}$, which are needed in (3.13), can be simplified considerably by using the following simple result:

Lemma 3.2. At $\alpha=\alpha_{0}$, the solution $u_{1}$ to (3.3b) with $u_{1}(1)=u_{1}(0)=u_{0}^{\prime}(1)$ is given, for any constant $D$, by

$$
u_{1}=\frac{\lambda_{1}}{3 \lambda_{0}}\left(1+u_{0}\right)+D u_{0 \alpha}
$$

Moreover, the correction term of order $\mathcal{O}(\delta)$ in the expansion (3.13) of the fold point is independent of $D$.

The proof is by a direct calculation. Clearly $u_{1}$ solves (3.3b) at $\alpha=\alpha_{0}$ since

$$
\begin{aligned}
\mathcal{L} u_{1}=\frac{\lambda_{1}}{3 \lambda_{0}} \mathcal{L}\left(1+u_{0}\right)=\frac{\lambda_{1}}{3 \lambda_{0}}\left[u_{0}^{\prime \prime}+\frac{2 \lambda_{0}}{\left(1+u_{0}\right)^{2}}\right] & =\frac{\lambda_{1}}{3 \lambda_{0}}\left[\frac{\lambda_{0}}{\left(1+u_{0}\right)^{2}}+\frac{2 \lambda_{0}}{\left(1+u_{0}\right)^{2}}\right] \\
& =\frac{\lambda_{1}}{\left(1+u_{0}\right)^{2}} .
\end{aligned}
$$

In addition, since $u_{0}(1)=0$, then $u_{1}(1)=\lambda_{1} / 3 \lambda_{0}=u_{0}^{\prime}(1)$ from (3.17), as required by (3.9). Finally, a tedious but direct computation using (3.18), (3.19), and (3.20), 
shows that $\lambda_{2}-\lambda_{1 \alpha}^{2} /\left[2 \lambda_{0 \alpha \alpha}\right]$ at $\alpha=\alpha_{0}$ is independent of the constant $D$ in (3.21). Therefore, the fold point correction is independent of the normalization of $u_{1}$. The details of this latter calculation are left to the reader.

Therefore, $D=0$ is taken to get $u_{1}=\lambda_{1}\left(1+u_{0}\right) /\left(3 \lambda_{0}\right)$. Upon substitution of $u_{1}$ into (3.19), it is observed that the integral term on the right-hand side of (3.19) vanishes identically. Then, using (3.16) for $I$, the following compact formula is obtained at $\alpha=\alpha_{0}$ :

$$
\lambda_{1 \alpha}=3 \lambda_{0} u_{0 \alpha}^{\prime}(1) .
$$

Reassuringly, this agrees with differentiation of (3.12) by $\alpha$ followed by evaluation at $\alpha_{0}$. Similarly, in (3.20) for $\lambda_{2}$, one sets $u_{1}=\lambda_{1}\left(1+u_{0}\right) /\left(3 \lambda_{0}\right)$ and $u_{2}(1)=u_{1}^{\prime}(1)=$ $\lambda_{1} u_{0}^{\prime}(1) /\left(3 \lambda_{0}\right)$, to obtain

$$
\lambda_{2} I=-\frac{2 \lambda_{1}}{3 \lambda_{0}} u_{0}^{\prime}(1) u_{0 \alpha}^{\prime}(1)+\frac{\lambda_{1}^{2}}{3 \lambda_{0}} I-\int_{0}^{1} u_{0 \alpha} u_{0}^{\prime \prime \prime \prime} d x .
$$

Expression (3.23) can be reduced further by integrating twice by parts as follows:

$$
\begin{aligned}
\int_{0}^{1} u_{0 \alpha} u_{0}^{\prime \prime \prime \prime} d x & =-\int_{0}^{1} u_{0 \alpha}^{\prime} u_{0}^{\prime \prime \prime} d x=-\left.u_{0 \alpha}^{\prime} u_{0}^{\prime \prime}\right|_{0} ^{1}+\int_{0}^{1} u_{0 \alpha}^{\prime \prime} u_{0}^{\prime \prime} d x \\
& =-2 u_{0 \alpha}^{\prime}(1) \lambda_{0}+\int_{0}^{1}\left(-\frac{2 \lambda_{0} u_{0 \alpha}}{\left(1+u_{0}\right)^{3}}\right)\left(\frac{\lambda_{0}}{\left(1+u_{0}\right)^{2}}\right) d x \\
& =-2 \lambda_{0} u_{0 \alpha}^{\prime}(1)-2 \lambda_{0}^{2} \int_{0}^{1} \frac{u_{0 \alpha}}{\left(1+u_{0}\right)^{5}} d x .
\end{aligned}
$$

Combining this last expression with (3.23) together with the formula for $I$ in (3.16) and $\lambda_{1}=3 \lambda_{0} u_{0}^{\prime}(1)$, it follows at $\alpha=\alpha_{0}$ that

$$
\lambda_{2}=6 \lambda_{0}\left[u_{0}^{\prime}(1)\right]^{2}-3 \lambda_{0}^{2}-\frac{3 \lambda_{0}^{3}}{u_{0 \alpha}^{\prime}(1)} \int_{0}^{1} \frac{u_{0 \alpha}}{\left(1+u_{0}\right)^{5}} d x .
$$

The results of the preceding calculations are summarized in the following statement:

Principal Result 3.3. Let $\alpha_{0}, \lambda_{0 c} \equiv \lambda_{0}\left(\alpha_{0}\right)$ be the location of the fold point at the end of the minimal solution branch for (3.3a) with boundary conditions $u_{0}(0)=$ $u_{0}(1)=0$. Then, for the singularly perturbed problem (3.1), a three-term expansion for the perturbed fold point location is

$$
\lambda_{c}=\lambda_{0 c}+3 \lambda_{0} \delta^{1 / 2} u_{0}^{\prime}(1)+\delta \hat{\lambda}_{2}+\cdots, \quad \hat{\lambda}_{2} \equiv \lambda_{2}\left(\alpha_{0}\right)-\frac{\lambda_{1 \alpha}^{2}\left(\alpha_{0}\right)}{2 \lambda_{0 \alpha \alpha}\left(\alpha_{0}\right)} .
$$

Here $\hat{\lambda}_{2}$ is defined in terms of $u_{0}$ and $u_{0 \alpha}$ by

$$
\hat{\lambda}_{2}=6 \lambda_{0}\left[u_{0}^{\prime}(1)\right]^{2}-3 \lambda_{0}^{2}-\frac{3 \lambda_{0}^{3}}{u_{0 \alpha}^{\prime}(1)} \int_{0}^{1} \frac{u_{0 \alpha}}{\left(1+u_{0}\right)^{5}} d x-\frac{\left[u_{0 \alpha}^{\prime}(1)\right]^{3}}{2}\left(\int_{0}^{1} \frac{u_{0 \alpha}^{3}}{\left(1+u_{0}\right)^{4}} d x\right)^{-1} .
$$


For the unit slab, the minimal solution branch for the unperturbed problem (3.3a) can be obtain implicitly in terms of the parameter $\alpha \equiv u_{0}(1 / 2)$. Multiply (3.3a) by $u_{0}^{\prime}$ and integrate once to obtain

$$
u_{0}^{\prime}=\sqrt{\frac{2 \lambda_{0}}{1+\alpha}}\left(\frac{u-\alpha}{u+1}\right)^{1 / 2} .
$$

A further integration using $u_{0}(1 / 2)=\alpha$ and $u_{0}(1)=0$, determines $\lambda_{0}(\alpha)$ as

$$
\begin{aligned}
\lambda_{0}(\alpha) & =2(1+\alpha)\left[2 \int_{\sqrt{1+\alpha}}^{1} \frac{s^{2} d s}{\sqrt{s^{2}-(1+\alpha)}}\right]^{2} \\
& =2(1+\alpha)\left[\sqrt{-\alpha}+(1+\alpha) \log \left(\frac{1+\sqrt{-\alpha}}{\sqrt{1+\alpha}}\right)\right]^{2} .
\end{aligned}
$$

Upon setting $\lambda_{0 \alpha}=0$, the fold point $\alpha_{0} \approx-0.389$ and $\lambda_{0 c} \approx 1.400$ is determined. By using this solution the various terms needed in (3.25) are easily calculated. In this way, (3.25) leads to the following explicit three-term expansion valid for $\delta \ll 1$ :

$$
\lambda_{c}=1.400+5.600 \delta^{1 / 2}+25.451 \delta+\cdots .
$$

In Fig. 6(b) a comparison of the two-term and three-term asymptotic results for $\lambda_{c}$ versus $\delta$ from (3.28) is provided alongside the corresponding full numerical result computed from (1.1). The three-term approximation in (3.28) is seen to provide a reasonably accurate determination of $\lambda_{c}$. For $\delta=0.01$, in Fig. 6(a) the two-term approximation (3.12) is compared with the global bifurcation curve with the full numerical result computed from (1.1) and from the membrane MEMS problem (1.2), corresponding to $\delta=0$. It is clear that the fold point location depends rather sensitively on $\delta$, even when $\delta \ll 1$, owing to the $\mathcal{O}\left(\delta^{1 / 2}\right)$ limiting behavior.

4. Biharmonic Nonlinear Eigenvalue Problem: Multidimensional Domain. The results of $\S 3.1$ are now extended to the case of a bounded two-dimensional domain $\Omega$ with smooth boundary $\partial \Omega$. Equation (1.1) is considered in the limit $\delta \rightarrow 0$, and it is assumed that the fold point location at the end of the minimal solution branch $u_{0}(x, \alpha), \lambda_{0}(\alpha)$ for the unperturbed problem

$$
\Delta u_{0}=\frac{\lambda_{0}}{\left(1+u_{0}\right)^{2}}, \quad x \in \Omega ; \quad u_{0}=0, \quad x \in \partial \Omega,
$$

has been determined. This fold point location is labeled as $\lambda_{0 c}=\lambda_{0}\left(\alpha_{0}\right)$ for some $\alpha=\alpha_{0}$. For an arbitrary domain $\Omega, \alpha$ can be chosen to be the $L_{2}$ norm of $u_{0}$. For the unit disk, where $u_{0}(r)$ is radially symmetric, it is more convenient to define $\alpha$ by $\alpha=u_{0}(0)$.

For the perturbed problem (1.1), $\lambda$ and the outer solution for $u$ are expanded in powers of $\delta^{1 / 2}$ as in (3.2), to obtain

$$
\begin{aligned}
& \mathcal{L} u_{1} \equiv \Delta u_{1}+\frac{2 \lambda_{0}}{\left(1+u_{0}\right)^{3}} u_{1}=\frac{\lambda_{1}}{\left(1+u_{0}\right)^{2}}, \quad x \in \Omega, \\
& \mathcal{L} u_{2}=\frac{\lambda_{2}}{\left(1+u_{0}\right)^{2}}-\frac{2 \lambda_{1} u_{1}}{\left(1+u_{0}\right)^{3}}+\frac{3 \lambda_{0} u_{1}^{2}}{\left(1+u_{0}\right)^{4}}+\Delta^{2} u_{0}, \quad x \in \Omega .
\end{aligned}
$$

The expansion of the perturbed fold point location is again as given in (3.13). 


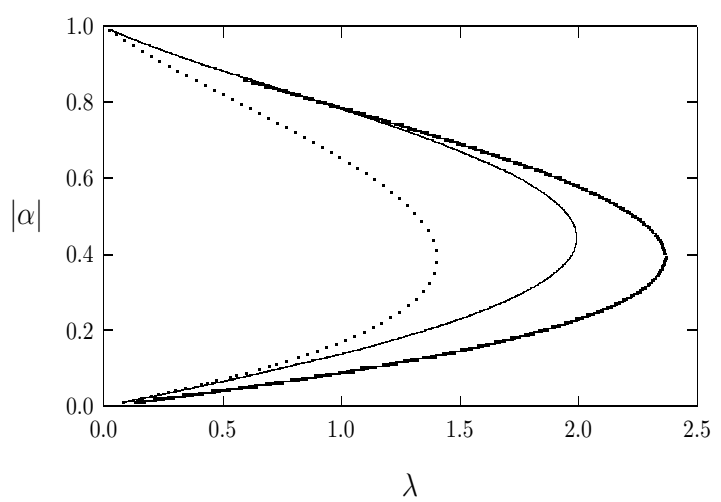

(a) $|\alpha|$ vs. $\lambda$ (Unit Slab)

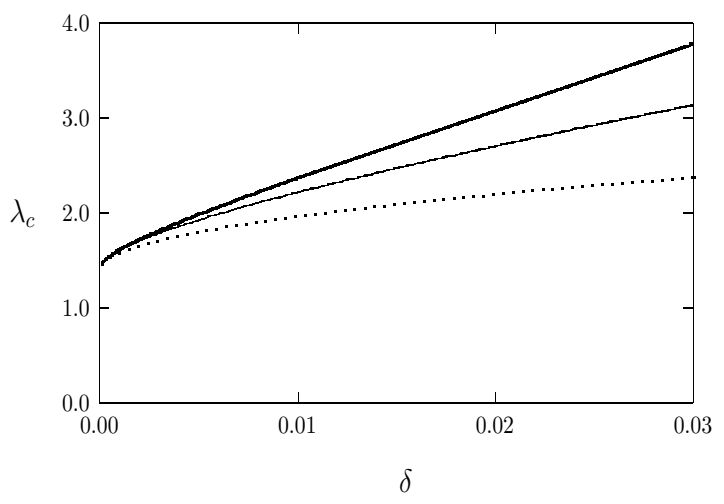

(b) $\lambda_{c}$ vs. $\delta$ (Unit Slab)

Fig. 6. Left figure: Plot of numerically computed global bifurcation diagram $|\alpha|=|u(1 / 2)|$ versus $\lambda$ for (1.1) with $\delta=0.01$ (heavy solid curve) compared to the two-term asymptotic result (3.12) (solid curve) and the unperturbed $\delta=0$ membrane MEMS result from (1.2) (dashed curve). Right figure: Comparison of numerically computed fold point $\lambda_{c}$ versus $\delta$ (heavy solid curve) with the two-term (dashed curve) and the three-term (solid curve) asymptotic result from (3.28).

To derive boundary conditions for $u_{1}$ and $u_{2}$, a boundary layer solution near $\partial \Omega$ with width $\mathcal{O}\left(\delta^{1 / 2}\right)$ is constructed. It is advantageous to implement an orthogonal coordinate system $\eta, s$, where $\eta>0$ measures the perpendicular distance from $x \in \Omega$ to $\partial \Omega$, whereas on $\partial \Omega$ the coordinate $s$ denotes arclength. In terms of $(\eta, s),(1.1)$ transforms to

$$
\begin{aligned}
& -\delta\left(\partial_{\eta \eta}-\frac{\kappa}{1-\kappa \eta} \partial_{\eta}+\frac{1}{1-\kappa \eta} \partial_{s}\left(\frac{1}{1-\kappa \eta} \partial_{s}\right)\right)^{2} u \\
& \quad+\left(\partial_{\eta \eta} u-\frac{\kappa}{1-\kappa \eta} \partial_{\eta} u+\frac{1}{1-\kappa \eta} \partial_{s}\left(\frac{1}{1-\kappa \eta} \partial_{s} u\right)\right)=\frac{\lambda}{(1+u)^{2}} .
\end{aligned}
$$

Here $\kappa=\kappa(s)$ is the curvature of $\partial \Omega$, with $\kappa=1$ for the unit disk. The inner variables and the inner expansion, defined in an $\mathcal{O}\left(\delta^{1 / 2}\right)$ neighborhood of $\partial \Omega$, are 
then introduced as

$$
\hat{\eta}=\eta / \delta^{1 / 2}, \quad u=\delta^{1 / 2} v, \quad v=v_{0}+\delta^{1 / 2} v_{1}+\delta v_{2}+\cdots .
$$

After substituting (4.4) into (4.3) and collecting powers of $\delta$, some lengthy but straightforward algebra produces the following sequence of problems on $-\infty<\hat{\eta}<0$ :

$$
\begin{aligned}
&-v_{0 \hat{\eta} \hat{\eta} \hat{\eta} \hat{\eta}}+v_{0 \hat{\eta} \hat{\eta}}=0, \quad v_{0}=v_{0 \hat{\eta}}=0, \quad \text { on } \quad \hat{\eta}=0 \\
&-v_{1 \hat{\eta} \hat{\eta} \hat{\eta} \hat{\eta}}+v_{1 \hat{\eta} \hat{\eta}}=-2 \kappa v_{0 \hat{\eta} \hat{\eta} \hat{\eta}}+\kappa v_{0 \hat{\eta}}+\lambda_{0}, \quad v_{1}=v_{1 \hat{\eta}}=0, \quad \text { on } \quad \hat{\eta}=0 \\
&-v_{2 \hat{\eta} \hat{\eta} \hat{\eta} \hat{\eta}}+v_{2 \hat{\eta} \hat{\eta}}=-2 \kappa v_{1 \hat{\eta} \hat{\eta} \hat{\eta}}+\kappa v_{1 \hat{\eta}}-2 \kappa^{2} \hat{\eta} v_{0 \hat{\eta} \hat{\eta} \hat{\eta}}-\kappa^{2} v_{0 \hat{\eta} \hat{\eta}}+\kappa^{2} \hat{\eta} v_{0 \hat{\eta}} \\
& \quad+2 v_{0 \hat{\eta} \hat{\eta} s s}-v_{0 s s}+\lambda_{1}-2 \lambda_{0} v_{0}, \quad v_{2}=v_{2 \hat{\eta}}=0, \quad \text { on } \quad \hat{\eta}=0 .
\end{aligned}
$$

The asymptotic behavior of the solution to (4.5) with no exponential growth as $\eta \rightarrow$ $-\infty$ is given in terms of unknown functions $c_{0}(s), c_{1}(s)$, and $c_{2}(s)$ by

$$
v_{0} \sim-c_{0}+c_{0} \hat{\eta}, \quad v_{1} \sim-c_{1}+\left(c_{1}-\frac{c_{0} \kappa}{2}\right) \hat{\eta}
$$

with $v_{2} \sim-c_{2}+\mathcal{O}(\eta)$ as $\eta \rightarrow 0$. Therefore, with $u=\delta^{1 / 2} v$, and by rewriting $v$ in terms of the outer variable $\eta=\hat{\eta} \delta^{1 / 2}$, the following matching condition, analogous to (3.8), is obtained for the outer solution:

$$
u \sim c_{0} \eta+\delta^{1 / 2}\left[-c_{0}+\eta\left(c_{1}-\frac{c_{0} \kappa}{2}\right)\right]+\delta\left[-c_{1}+\mathcal{O}(\eta)\right]+\cdots .
$$

Noting that the outer normal derivative $\partial_{n} u$ on $\partial \Omega$ is simply $\partial_{n} u=-\partial_{\eta} u,(4.6)$ then implies the following boundary conditions for the outer solutions $u_{1}$ and $u_{2}$ in $(4.2)$ :

$$
u_{0}=0, \quad u_{1}=\partial_{n} u_{0}, \quad u_{2}=\partial_{n} u_{1}+\frac{\kappa}{2} \partial_{n} u_{0}, \quad x \in \partial \Omega
$$

The functions $c_{0}(s)$ and $c_{1}(s)$, which determine the leading two boundary layers solutions explicitly, are given by

$$
c_{0}=-\partial_{n} u_{0}, \quad c_{1}=-\partial_{n} u_{1}-\frac{\kappa}{2} \partial_{n} u_{0}, \quad x \in \partial \Omega
$$

with a more complicated expression, which we omit, for $c_{2}(s)$. Notice that the boundary condition for $u_{2}$ on $\partial \Omega$ depends on the curvature $\kappa$ of $\partial \Omega$.

The remainder of the analysis to calculate the terms in the expansion of the fold point is similar to that in $\S 3$. At $\alpha=\alpha_{0}, \mathcal{L} u_{0 \alpha}=0$, and so each of the problems in (4.2) must satisfy a solvability condition. By applying Green's identity to $u_{0 \alpha}$ and $u_{1}$, together with the boundary condition $u_{1}=\partial_{n} u_{0}$ on $\partial \Omega$, it follows at $\alpha=\alpha_{0}$ that

$$
\lambda_{1} I=-\int_{\partial_{\Omega}}\left(\partial_{n} u_{0}\right)\left(\partial_{n} u_{0 \alpha}\right) d x, \quad I \equiv \int_{\Omega} \frac{u_{0 \alpha}}{\left(1+u_{0}\right)^{2}} d x .
$$

The integral $I$ can be written more conveniently by using the following lemma:

LEMMA 4.1. At $\alpha=\alpha_{0}$, the following identity holds:

$$
I \equiv \int_{\Omega} \frac{u_{0 \alpha}}{\left(1+u_{0}\right)^{2}} d x=-\frac{1}{3 \lambda_{0}} \int_{\partial \Omega} \partial_{n} u_{0 \alpha} d x .
$$


To prove this result, the equation for $u_{0 \alpha}$ together with Green's second identity and the divergence theorem is used to calculate

$$
\begin{aligned}
I=-\frac{1}{2 \lambda_{0}} \int_{\Omega}\left(1+u_{0}\right) \Delta u_{0 \alpha} d x & =-\frac{1}{2 \lambda_{0}}\left[\int_{\partial \Omega} \partial_{n} u_{0 \alpha} d x+\int_{\Omega} u_{0 \alpha} \Delta u_{0} d x\right] \\
& =-\frac{1}{2 \lambda_{0}} \int_{\partial \Omega} \partial_{n} u_{0 \alpha} d x-\frac{I}{2} .
\end{aligned}
$$

Solving for $I$ then gives the result.

Upon substituting (4.9) into (4.8), $\lambda_{1}$ can be expressed at $\alpha=\alpha_{0}$ as

$$
\lambda_{1}=3 \lambda_{0}\left(\frac{\int_{\partial \Omega}\left(\partial_{n} u_{0}\right)\left(\partial_{n} u_{0 \alpha}\right) d x}{\int_{\partial \Omega} \partial_{n} u_{0 \alpha} d x}\right) .
$$

From (3.13) this then specifies the correction of order $\mathcal{O}\left(\delta^{1 / 2}\right)$ to the fold point location.

To determine the $\mathcal{O}(\delta)$ term in the expansion (3.13) of the fold point, the terms $\lambda_{0 \alpha \alpha}, \lambda_{1 \alpha}$, and $\lambda_{2}$ at $\alpha=\alpha_{0}$ must be calculated. This is done through solvability conditions with $u_{0 \alpha}$ in a similar way as in $\S 3$. This procedure leads to the following identities at $\alpha=\alpha_{0}$ :

$$
\begin{aligned}
\lambda_{0 \alpha \alpha}= & \frac{18 \lambda_{0}^{2}}{\left(\int_{\partial \Omega} \partial_{n} u_{0 \alpha} d x\right)} \int_{\Omega} \frac{u_{0 \alpha}^{3}}{\left(1+u_{0}\right)^{4}} d x \\
\lambda_{1 \alpha} I= & -\int_{\Omega} u_{0 \alpha}\left(\frac{6 \lambda_{0} u_{1} u_{0 \alpha}}{\left(1+u_{0}\right)^{4}}-\frac{2 \lambda_{1} u_{0 \alpha}}{\left(1+u_{0}\right)^{3}}\right) d x-\int_{\partial \Omega}\left[\partial_{n} u_{0 \alpha}\right]^{2} d x \\
\lambda_{2} I=- & \int_{\Omega} u_{0 \alpha}\left[\frac{3 \lambda_{0} u_{1}^{2}}{\left(1+u_{0}\right)^{4}}-\frac{2 \lambda_{1} u_{1}}{\left(1+u_{0}\right)^{3}}+\Delta^{2} u_{0}\right] d x \\
& -\int_{\partial \Omega}\left[\partial_{n} u_{1}+\frac{\kappa}{2} \partial_{n} u_{0}\right] \partial_{n} u_{0 \alpha} d x
\end{aligned}
$$

In contrast to the one-dimensional case of $\S 3, u_{1}$ cannot be obtained as explicitly as in Lemma 3.2. In place of Lemma 3.2, it is readily shown that $u_{1}$ admits the following decomposition at $\alpha=\alpha_{0}$ :

$$
u_{1}=\frac{\lambda_{1}}{3 \lambda_{0}}\left(1+u_{0}\right)+u_{1 a}+D u_{0 \alpha}
$$

Here $D$ is any scalar constant, and $u_{1 a}$ at $\alpha=\alpha_{0}$ is the unique solution to

$$
\mathcal{L} u_{1 a}=0, \quad x \in \Omega ; \quad u_{1 a}=\partial_{n} u_{0}-\frac{\lambda_{1}}{3 \lambda_{0}}, \quad x \in \partial \Omega ; \quad \int_{\Omega} u_{1 a} u_{0 \alpha} d x=0 .
$$

By substituting (4.12) into (4.11), a straightforward calculation shows that $\lambda_{2}-$ $\lambda_{1 \alpha}^{2} /\left[2 \lambda_{0 \alpha \alpha}\right]$ is independent of $D$. Hence, set $D=0$ in (4.12) for simplicity. By substituting (4.12) for $u_{1}$ into (4.11b), and then using (4.9) for $I, \lambda_{1 \alpha}$ at $\alpha=\alpha_{0}$ can be written as

$$
\lambda_{1 \alpha}=\frac{18 \lambda_{0}^{2}}{\left(\int_{\partial \Omega} \partial_{n} u_{0 \alpha} d x\right)} \int_{\Omega} \frac{u_{1 a} u_{0 \alpha}^{3}}{\left(1+u_{0}\right)^{4}} d x+3 \lambda_{0}\left(\frac{\int_{\partial \Omega}\left[\partial_{n} u_{0 \alpha}\right]^{2} d x}{\int_{\partial \Omega} \partial_{n} u_{0 \alpha} d x}\right)
$$


Similarly, equation (4.12) for $u_{1}$ and (4.9) for $I$ are substituted into (4.11c). In addition, in the resulting expression, the following identity which is readily derived by integration by parts, is used:

$$
\int_{\Omega} u_{0 \alpha} \Delta^{2} u_{0} d x=-2 \lambda_{0}^{2} \int_{\Omega} \frac{u_{0 \alpha}}{\left(1+u_{0}\right)^{5}} d x-\lambda_{0} \int_{\partial \Omega} \partial_{n} u_{0 \alpha} d x
$$

In this way, expression (4.11c) for $\lambda_{2}$ at $\alpha=\alpha_{0}$ simplifies to

$$
\begin{aligned}
\lambda_{2}= & \frac{2 \lambda_{1}^{2}}{3 \lambda_{0}}-3 \lambda_{0}^{2}+\frac{3 \lambda_{0}}{\left(\int_{\partial \Omega} \partial_{n} u_{0 \alpha} d x\right)} \int_{\partial \Omega}\left[\partial_{n} u_{1 a}+\frac{\kappa}{2} \partial_{n} u_{0}\right] \partial_{n} u_{0 \alpha} d x \\
& \quad-\frac{6 \lambda_{0}^{3}}{\left(\int_{\partial \Omega} \partial_{n} u_{0 \alpha} d x\right)} \int_{\Omega} \frac{u_{0 \alpha}}{\left(1+u_{0}\right)^{5}} d x+\frac{9 \lambda_{0}^{2}}{\left(\int_{\partial \Omega} \partial_{n} u_{0 \alpha} d x\right)} \int_{\Omega} \frac{u_{1 a}^{2} u_{0 \alpha}}{\left(1+u_{0}\right)^{4}} d x .
\end{aligned}
$$

The results of the preceding calculations are summarized as follows:

Principal Result 4.2. Let $\lambda_{c} \equiv \lambda_{0}\left(\alpha_{0}\right)$ be the fold point location at the end of the minimal solution branch for the unperturbed problem (4.1) in a bounded twodimensional domain $\Omega$, with smooth boundary $\partial \Omega$. Then, for (1.1) with $\delta \ll 1$, a three-term expansion for the perturbed fold point location is

$$
\begin{aligned}
& \lambda_{c}=\lambda_{0 c}+3 \lambda_{0} \delta^{1 / 2}\left(\frac{\int_{\partial \Omega}\left(\partial_{n} u_{0}\right)\left(\partial_{n} u_{0 \alpha}\right) d x}{\int_{\partial \Omega} \partial_{n} u_{0 \alpha} d x}\right)+\delta \hat{\lambda}_{2}+\cdots, \\
& \hat{\lambda}_{2} \equiv \lambda_{2}\left(\alpha_{0}\right)-\frac{\lambda_{1 \alpha}^{2}\left(\alpha_{0}\right)}{2 \lambda_{0 \alpha \alpha}\left(\alpha_{0}\right)} .
\end{aligned}
$$

Here $\lambda_{0 \alpha \alpha}\left(\alpha_{0}\right), \lambda_{1 \alpha}\left(\alpha_{0}\right)$, and $\lambda_{2}\left(\alpha_{0}\right)$ are as given in (4.11a), (4.14), and (4.16), respectively.

For the special case of the unit disk where $\Omega:=\{x|| x \mid \leq 1\}$, then $u_{0}=u_{0}(r)$ and $u_{0 \alpha}=u_{0 \alpha}(r)$ are radially symmetric, and $\kappa=1$. Therefore, for this special geometry, $u_{1 a} \equiv 0$ from (4.13), and consequently the various terms in (4.17) can be simplified considerably. In analogy with Principal Result 3.3, the following asymptotic expansion is obtained for the fold point location of (1.1) in the limit $\delta \rightarrow 0$ for the unit disk:

Corollary 4.3. For the special case of the unit disk, let $\alpha_{0}=u_{0}(0)$ and $\lambda_{0 c} \equiv$ $\lambda_{0}\left(\alpha_{0}\right)$ be the location of the fold point at the end of the minimal radially symmetric solution branch for the unperturbed problem (4.1). Then, for (1.1) with $\delta \ll 1$, a three-term expansion for the perturbed fold point location is

$$
\lambda_{c}=\lambda_{0 c}+3 \lambda_{0} \delta^{1 / 2} u_{0}^{\prime}(1)+\delta \hat{\lambda}_{2}+\cdots, \quad \hat{\lambda}_{2} \equiv \lambda_{2}\left(\alpha_{0}\right)-\frac{\lambda_{1 \alpha}^{2}\left(\alpha_{0}\right)}{2 \lambda_{0 \alpha \alpha}\left(\alpha_{0}\right)} .
$$

Here $\hat{\lambda}_{2}$ is defined in terms of $u_{0}$ and $u_{0 \alpha}$ by

$$
\begin{gathered}
\hat{\lambda}_{2}=\frac{3}{2} \lambda_{0} u_{0}^{\prime}(1)+6 \lambda_{0}\left[u_{0}^{\prime}(1)\right]^{2}-3 \lambda_{0}^{2}-\frac{6 \lambda_{0}^{3}}{u_{0 \alpha}^{\prime}(1)} \int_{0}^{1} \frac{r u_{0 \alpha}}{\left(1+u_{0}\right)^{5}} d r \\
-\frac{\left[u_{0 \alpha}^{\prime}(1)\right]^{3}}{2}\left(\int_{0}^{1} \frac{r u_{0 \alpha}^{3}}{\left(1+u_{0}\right)^{4}} d x\right)^{-1} .
\end{gathered}
$$

The first term in $\hat{\lambda}_{2}$ above arises from the constant curvature of $\partial \Omega$. 
For the unit disk, numerical values for the coefficients in the expansion (4.18) are obtained by first using COLSYS [1] to solve for $u_{0}$ and $u_{0 \alpha}$. In this way, the explicit three-term expansion for the unit disk is

$$
\lambda_{c}=0.789+1.578 \delta^{1 / 2}+6.261 \delta+\cdots .
$$

In addition, for the unit disk it follows as in (3.12) that the global bifurcation diagram is given for $\delta \ll 1$ by

$$
\lambda \sim \lambda_{0}(\alpha)+3 \lambda_{0}(\alpha) u_{0}^{\prime}(1, \alpha) \delta^{1 / 2}+\mathcal{O}(\delta) .
$$

For the unit disk, Fig. 7(b) provides a comparison of the two-term and threeterm asymptotic results for $\lambda_{c}$ versus $\delta$ from (4.19) along with the corresponding full numerical result computed from (1.1). Since the coefficients in (4.19) are smaller than those in (3.28), the three-term approximation for the unit disk is seen to provide a more accurate determination of $\lambda_{c}$ than the result for the slab shown in Fig. 6(b). For $\delta=0.01$, Fig. 7(a) provides a comparison of the two-term approximation (4.20) to the global bifurcation curve with the full numerical result computed from (1.1) and also the membrane MEMS problem (1.2), corresponding to $\delta=0$. From this figure it is seen that (4.20) compares favorably with the full numerical result provided that $\alpha$ is not too close to -1 . Recall that $\lambda \rightarrow 4 / 9$ as $\alpha \rightarrow-1$ for (1.2), whereas from the numerical results in $\S 2, \lambda \rightarrow 0$ as $\alpha \rightarrow-1$ for (1.1) when $\delta>0$. The singular limit $\alpha \rightarrow-1$ is examined in the companion paper [17]

5. Perturbing from the Pure Biharmonic Eigenvalue Problem. Next, equation (1.1) is considered in the limit $\delta \gg 1$. To study this limiting case, (1.1) is rewritten as

$$
-\Delta^{2} u+\frac{1}{\delta} \Delta u=\frac{\tilde{\lambda}}{(1+u)^{2}}, \quad x \in \Omega ; \quad u=\partial_{n} u=0, \quad x \in \partial \Omega,
$$

where $\tilde{\lambda} \equiv \lambda / \delta$. For $\delta \gg 1$, the solution $u$ and the nonlinear eigenvalue parameter $\tilde{\lambda}$ are expanded as

$$
u=u_{0}+\frac{1}{\delta} u_{1}+\cdots, \quad \tilde{\lambda}=\tilde{\lambda}_{0}+\frac{1}{\delta} \tilde{\lambda}_{1}+\cdots .
$$

Inserting this expansion in (5.1) and collecting terms, requires that $u_{0}$ and $u_{1}$ satisfy

$$
\begin{aligned}
-\Delta^{2} u_{0} & =\frac{\tilde{\lambda}_{0}}{\left(1+u_{0}\right)^{2}}, \quad x \in \Omega ; \quad u_{0}=\partial_{n} u_{0}=0, \quad x \in \partial \Omega, \\
\mathcal{L}_{b} u_{1} & \equiv \Delta^{2} u_{1}-\frac{2 \tilde{\lambda}_{0}}{\left(1+u_{0}\right)^{3}} u_{1}=-\frac{\tilde{\lambda}_{1}}{\left(1+u_{0}\right)^{2}}+\Delta u_{0}, \quad x \in \Omega ; \\
u_{1} & =\partial_{n} u_{1}=0, \quad x \in \partial \Omega .
\end{aligned}
$$

The minimal solution branch for the unperturbed problem (5.3a) is parameterized as $\tilde{\lambda}_{0}(\alpha), u_{0}(x, \alpha)$. It is assumed that there is a simple fold point at the end of this branch with location $\alpha=\alpha_{0}$, where $\tilde{\lambda}_{0 c}=\tilde{\lambda}_{0}\left(\alpha_{0}\right)$. Since $\mathcal{L}_{b} u_{0 \alpha}=0$ at $\alpha=\alpha_{0}$, the solvability condition for $(5.3 \mathrm{~b})$ determines $\tilde{\lambda}_{1}$ at $\alpha=\alpha_{0}$ as

$$
I_{b} \tilde{\lambda}_{1}=\int_{\Omega} u_{0 \alpha} \Delta u_{0} d x, \quad I_{b}=\int_{\Omega} \frac{u_{0 \alpha}}{\left(1+u_{0}\right)^{2}} d x .
$$




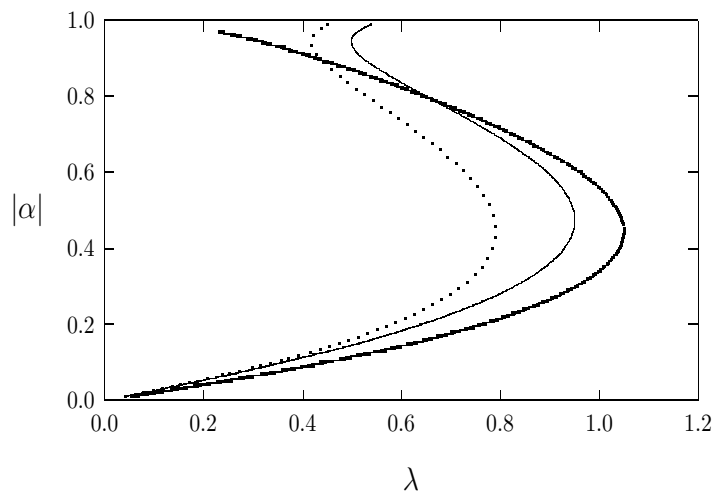

(a) $|\alpha|$ vs. $\lambda$ (Unit Disk)

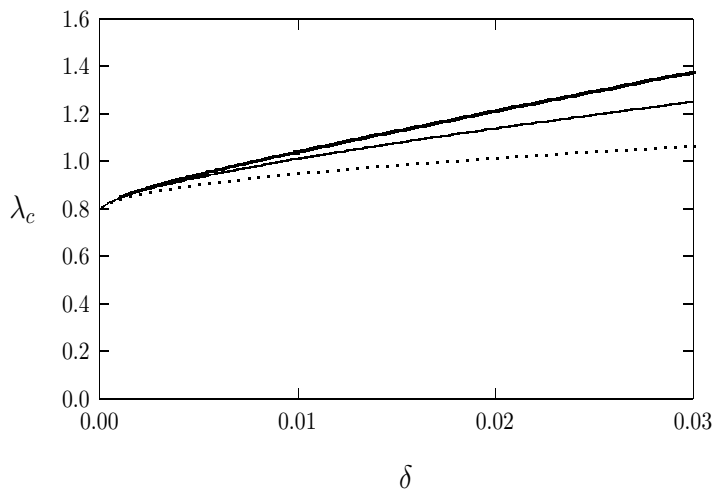

(b) $\lambda_{c}$ vs. $\delta$ (Unit Disk)

Fig. 7. Left figure: Plot of numerically computed global bifurcation diagram $|\alpha|=|u(0)|$ versus $\lambda$ for (1.1) with $\delta=0.01$ (heavy solid curve) compared to the two-term asymptotic result (4.20) (solid curve) and the unperturbed $\delta=0$ membrane MEMS result from (1.2) (dashed curve). Right figure: Comparison of numerically computed fold point $\lambda_{c}$ versus $\delta$ (heavy solid curve) with the two-term (dashed curve) and the three-term (solid curve) asymptotic result from (4.19).

By using the equation and boundary conditions for $u_{0 \alpha}$ and $u_{0}$, we can calculate $I_{b}$ using Green's identity as

$$
\begin{aligned}
I_{b}=\frac{1}{2 \lambda_{0}} \int_{\Omega}\left(u_{0}+1\right) \Delta^{2} u_{0 \alpha} d x & =\frac{1}{2 \lambda_{0}}\left[\int_{\partial \Omega} \partial_{n}\left(\Delta u_{0 \alpha}\right) d x+\int_{\Omega} u_{0 \alpha} \Delta^{2} u_{0} d x\right] \\
& =\frac{1}{2 \lambda_{0}} \int_{\partial \Omega} \partial_{n}\left(\Delta u_{0 \alpha}\right) d x-\frac{I_{b}}{2} .
\end{aligned}
$$

This yields that

$$
I_{b}=\frac{1}{3 \lambda_{0}} \int_{\partial \Omega} \partial_{n}\left(\Delta u_{0 \alpha}\right) d x
$$

PRINCIPAL RESUlT 5.1. Let $\tilde{\lambda}_{0}(\alpha)$ and $u_{0}(x, \alpha)$ be the minimal solution branch for the pure biharmonic problem (5.3a), and assume that there is a simple fold point 
at $\alpha=\alpha_{0}$ where $\tilde{\lambda}_{0 c}=\tilde{\lambda}_{0}\left(\alpha_{0}\right)$. Then, for $\delta \gg 1$, the expansion of the fold point for (1.1) is given by

$$
\lambda_{c} \sim \delta\left[\tilde{\lambda}_{0 c}+\delta^{-1} \tilde{\lambda}_{1}\left(\alpha_{0}\right)+\mathcal{O}\left(\delta^{-2}\right)\right], \quad \tilde{\lambda}_{1}\left(\alpha_{0}\right) \equiv 3 \lambda_{0}\left(\frac{\int_{\Omega} u_{0 \alpha} \Delta u_{0} d x}{\int_{\partial \Omega} \partial_{n}\left(\Delta u_{0 \alpha}\right) d x}\right) .
$$

For the special case of the unit disk or a slab domain of unit length, then $\tilde{\lambda}_{1}\left(\alpha_{0}\right)$ reduces to

$$
\begin{aligned}
\tilde{\lambda}_{1}\left(\alpha_{0}\right) & \equiv \frac{3 \lambda_{0}}{\left.\partial_{r}\left(\Delta u_{0 \alpha}\right)\right|_{r=1}} \int_{0}^{1} u_{0 \alpha}\left(r u_{0 r}\right)_{r} d r, \quad \text { (Unit Disk) } \\
\tilde{\lambda}_{1} & =\frac{3 \lambda_{0}}{2 u_{0 \alpha}^{\prime \prime \prime}(1)} \int_{0}^{1} u_{0 \alpha} u_{0}^{\prime \prime} d x \quad \text { (Unit Slab) } .
\end{aligned}
$$

For the slab and the unit disk, the numerical values of the coefficients in the expansion (5.7) are calculated by using COLSYS [1] to solve for $u_{0}$ and $u_{0 \alpha}$ at the fold point of the minimal branch for the pure Biharmomic problem (5.3a). In this way, the following is obtained for $\delta \gg 1$ :

$$
\begin{array}{lll}
\lambda_{c} \sim \delta\left[70.095+\frac{1}{\delta} 1.729+\cdots\right] & (\text { Unit Slab) } \\
\lambda_{c} \sim \delta\left[15.412+\frac{1}{\delta} 1.001+\cdots\right] & \text { (Unit Disk) }
\end{array}
$$

Although (5.8) was derived in the limit $\delta \gg 1$, in Fig. 8 it is shown, rather remarkably, that (5.8) is also in rather close agreement with the full numerical result for $\lambda_{c}$, computed from (1.1), even when $\delta<1$. Therefore, for the unit disk and the unit slab, the limiting approximations for $\lambda_{c}$ when $\delta \ll 1$ from (3.28) and (4.19), together with the $\delta \gg 1$ result (5.8), can be used to rather accurately predict the fold point $\lambda_{c}$ for (1.1) for a wide range of $\delta>0$.

6. The Fringing-Field and Annulus Problems. Next, the fringing-field problem (1.4) is considered in the limit $\delta \rightarrow 0$ in a two-dimensional domain $\Omega$ with smooth boundary $\partial \Omega$. Let $\alpha_{0}, \lambda_{0 c}=\lambda_{0}\left(\alpha_{0}\right)$ be the location of the fold point for the unperturbed problem (4.1). To determine the leading order correction to the fold point location for (1.4) when $\delta \ll 1$, the solution to (1.4) is expanded along the minimal solution branch as

$$
u=u_{0}+\delta u_{1}+\cdots, \quad \lambda=\lambda_{0}+\delta \lambda_{1}+\cdots .
$$

The problem for $u_{1}$ is obtained by substituting (6.1) into (1.4), which yields

$$
\mathcal{L} u_{1} \equiv \Delta u_{1}+\frac{2 \lambda_{0}}{\left(1+u_{0}\right)^{3}} u_{1}=\frac{\lambda_{1}}{\left(1+u_{0}\right)^{2}}+\lambda_{0} \frac{\left|\nabla u_{0}\right|^{2}}{\left(1+u_{0}\right)^{2}}, \quad x \in \Omega ; \quad u_{1}=0 \quad x \in \partial \Omega .
$$

Since $\mathcal{L} u_{0 \alpha}=0$ at $\alpha=\alpha_{0}$, the solvability condition for (6.2) at $\alpha=\alpha_{0}$ determines $\lambda_{1}$ at $\alpha=\alpha_{0}$ as

$$
\lambda_{1}\left(\alpha_{0}\right)=-\frac{\lambda_{0}}{I} \int_{\Omega} \frac{\left|\nabla u_{0}\right|^{2} u_{0 \alpha}}{\left(1+u_{0}\right)^{2}} d x, \quad I \equiv \int_{\Omega} \frac{u_{0 \alpha}}{\left(1+u_{0}\right)^{2}} d x
$$




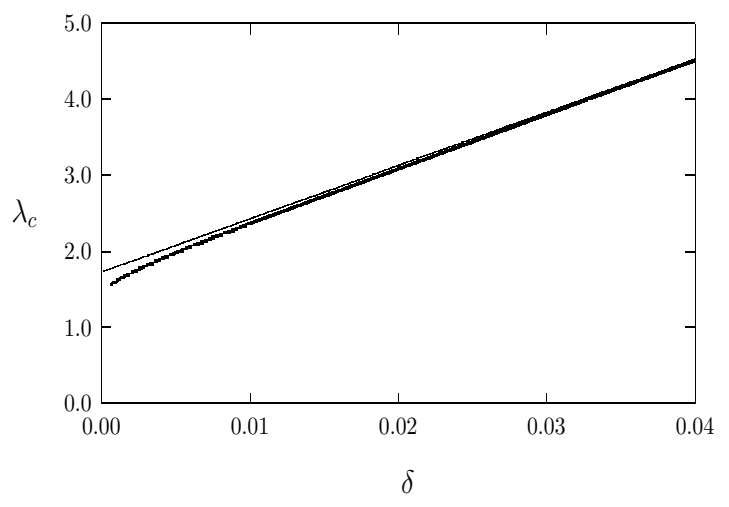

(a) $\lambda_{c}$ vs. $\delta$ (Unit Slab)

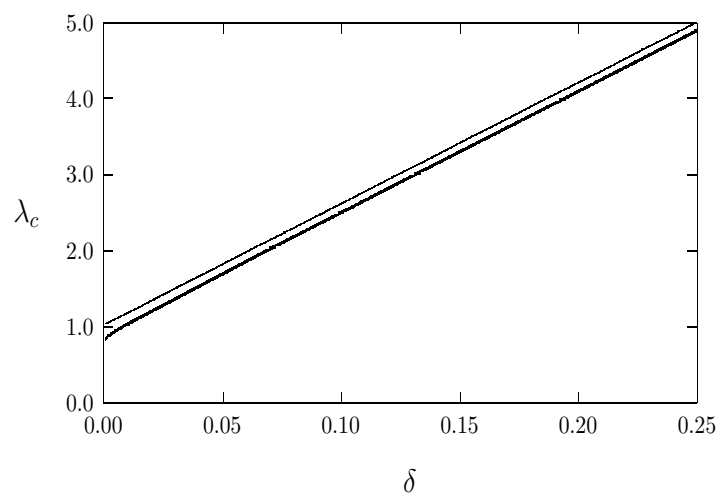

(b) $\lambda_{c}$ vs. $\delta$ (Unit Disk)

FIG. 8. Comparison of full numerical result for $\lambda_{c}$ versus $\delta$ (heavy solid curves) computed from (1.1) with the two-term asymptotic results (5.8) (solid curves) for the unit slab (left figure) and the unit disk (right figure).

where $I$ is given in Lemma 4.1. For the special case of the unit disk and the unit slab the result is summarized as follows:

Principal Result 6.1. Consider the fringing-field problem (1.4) with $\delta \ll 1$, and let $\lambda_{0 c}$ be the fold point location for the unperturbed problem (4.1). The, for $\delta \ll 1$, the fold point for the fringing-field problem (1.4) for the unit disk and a slab domain of unit length satisfies

$$
\begin{aligned}
& \lambda_{c} \sim \lambda_{0 c}+\frac{3 \lambda_{0}^{2} \delta}{u_{0 \alpha}^{\prime}(1)} \int_{0}^{1} \frac{r u_{0 r}^{2} u_{0 \alpha}}{\left(1+u_{0}\right)^{2}} d r, \quad \text { (Unit Disk) } \\
& \lambda_{c} \sim \lambda_{0 c}+\frac{3 \lambda_{0}^{2} \delta}{2 u_{0 \alpha}^{\prime}(1)} \int_{0}^{1} \frac{\left(u_{0}^{\prime}\right)^{2} u_{0 \alpha}}{\left(1+u_{0}\right)^{2}} d x . \quad \text { (Unit Slab) } .
\end{aligned}
$$

For the special case of the unit slab, as well as the unit disk considered in [21], the coefficient in (6.4) can be evaluated from the numerical solution of (4.1) to obtain

$$
\lambda_{c} \sim 0.789-0.160 \delta, \quad(\text { Unit Disk }) ; \quad \lambda_{c} \sim 1.400-0.529 \delta, \quad \text { (Unit Slab) } .
$$


Since the coefficients of $\delta$ are negative, the fringing field reduces the pull-in voltage. In Fig. 9(a) a very favorable comparison is shown between (6.5) for $\lambda_{c}$ in the unit disk and the full numerical result computed from (1.4). A similar comparison for the unit slab is shown in Fig. 9(b). Since the coefficients of $\delta$ in (6.5) are rather small, (6.5) provides a rather good prediction of $\lambda_{c}$ even for only moderately small $\delta$.

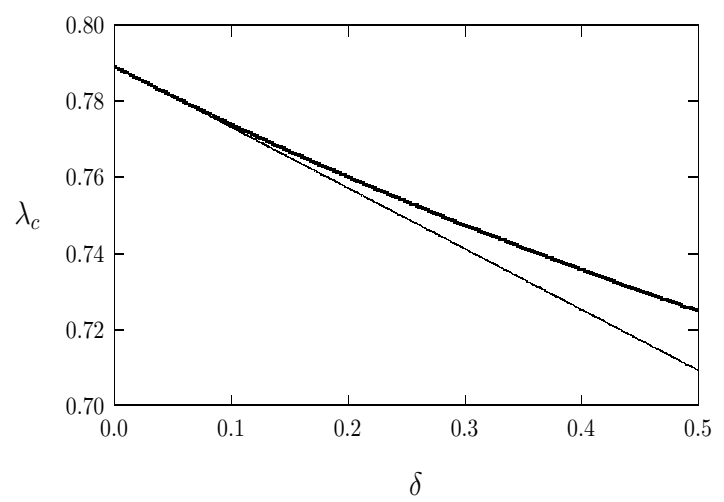

(a) $\lambda_{c}$ vs. $\delta$ (Unit Disk)

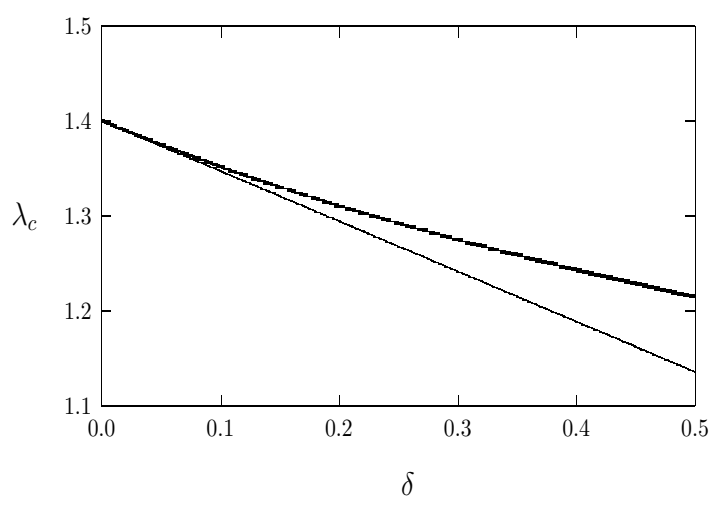

(b) $\lambda_{c}$ vs. $\delta$ (Unit Slab)

Fig. 9. Left figure: Comparison of full numerical result for $\lambda_{c}$ versus $\delta$ (heavy solid curve) for the fringing-field problem computed from (1.4) with the two-term asymptotic result (6.5) (solid curve) for the unit disk. Right figure: A similar comparison for the unit slab.

6.1. The Annulus Problem. Finally, the annulus problem (1.5) is considered in the limit $\delta \ll 1$ of a small inner radius. In this limit, (1.5) is singularly perturbed, and the construction of the solution requires the matching of an outer solution defined for $r=\mathcal{O}(1)$ to an appropriate inner solution defined near $r=\delta \ll 1$. Related nonlinear eigenvalue problems for Bratu's equation have been considered previously in [24] and [23]. Therefore, only a rather briefly outline of the singular perturbation analysis is given here.

Let $u_{0}(r, \alpha), \lambda_{0}(\alpha)$ be the radially symmetric minimal solution branch for the unperturbed problem (4.1) on $0 \leq r \leq 1$. For the perturbed problem (1.5), $\lambda$ and the 
outer solution $u$, valid for $r=\mathcal{O}(1)$, are expanded as

$$
u=u_{0}+\left(\frac{-1}{\log \delta}\right) u_{1}+\cdots, \quad \lambda=\lambda_{0}+\left(\frac{-1}{\log \delta}\right) \lambda_{1}+\cdots .
$$

From (1.5) it follows that $u_{1}$ satisfies

$$
\mathcal{L} u_{1} \equiv \Delta u_{1}+\frac{2 \lambda_{0}}{\left(1+u_{0}\right)^{3}} u_{1}=\frac{\lambda_{1}}{\left(1+u_{0}\right)^{2}}, \quad 0<r \leq 1 ; \quad u_{1}=0, \quad \text { on } \quad r=1 .
$$

The matching condition to the inner solution will then lead to a Coulomb singularity for $u_{1}$ as $r \rightarrow 0$, which then completes the specification of the problem for $u_{1}$.

In the inner region, defined for $r=\mathcal{O}(\delta)$, the inner variables $\rho=r / \delta$ and $u=$ $v /(-\log \delta)$ are introduced. To leading order, it follows from (1.5) that $\Delta v=0$ with $v(1)=0$. Thus, $v=A \log \rho$ for some unknown constant $A$. In terms of outer variables this yields the matching condition

$$
u \sim A+\left(\frac{-1}{\log \delta}\right) A \log r, \quad r \rightarrow 0 .
$$

The matching of the inner and outer solutions then determines $A=u_{0}(0)$, and that $u_{1}$ has the singular behavior

$$
u_{1} \sim u_{0}(0) \log r, \quad \text { as } \quad r \rightarrow 0 .
$$

The problem (6.7) with singular behavior (6.8) determines $u_{1}$ and $\lambda_{1}$. To determine $\lambda_{1}\left(\alpha_{0}\right)$ from a solvability condition, Green's identity is applied to $u_{0 \alpha}$ and $u_{1}$ over a punctured disk $\varepsilon<r<1$ in the limit $\varepsilon \rightarrow 0$. This yields that $\lambda_{1} I=-2 \pi u_{0}(0) u_{0 \alpha}(0)$, where $I$ is given in Lemma 4.1. The result is summarized as follows:

Principal Result 6.2. Consider the annulus problem (1.4) with $\delta \ll 1$, and let $\lambda_{0 c}$ be the fold point location at the end of the minimal solution branch for the unperturbed problem (4.1). The, for $\delta \ll 1$, the fold point for (1.5) satisfies

$$
\lambda_{c} \sim \lambda_{0 c}+\left(\frac{-1}{\log \delta}\right) \frac{3 \lambda_{0} u_{0}(0) u_{0 \alpha}(0)}{u_{0 \alpha}^{\prime}(1)}+\mathcal{O}\left(\frac{-1}{\log \delta}\right)^{2} .
$$

With the parameterization $u_{0}(0)=\alpha$ for the unperturbed solution, the terms in (6.9) are calculated from the numerical solution of the unperturbed problem (4.1). This yields the explicit two-term expansion

$$
\lambda_{c} \sim 0.789+1.130\left(\frac{-1}{\log \delta}\right)++\mathcal{O}\left(\frac{-1}{\log \delta}\right)^{2} .
$$

Owing to the logarithmic dependence on $\delta$, the fold point location experiences a rather large perturbation even for $\delta$ rather small. This was observed in Fig. 4(b).

7. Conclusion. Asymptotic expansions for the fold point location at the end of the minimal solution branch for (1.1) were calculated in the limiting parameter range $\delta \ll 1$ and $\delta \gg 1$ for an arbitrary domain $\Omega$ with smooth boundary. In addition, two-term asymptotic approximations for the fold point location of the fringing-field (1.4) and annulus problems (1.5) were derived for small $\delta$. The coefficients in these asymptotic expansions were evaluated numerically for both the unit slab and the unit 
disk. The results can be used to determine the shift in the pull-in voltage when the basic membrane problem (1.2) is perturbed to either (1.1), (1.4), or (1.5).

In the companion paper [17] an asymptotic theory describing the destruction of the infinite fold points for (1.2) when the disk problem (1.2) is perturbed for $0<\delta \ll 1$ to either the biharmonic problem (1.1), the fringing-field problem (1.4), or the annulus problems (1.5), is given by constructing the limiting form of the bifurcation diagram when $|u|_{\infty}=1-\varepsilon$, where $\varepsilon \rightarrow 0^{+}$. Moreover, in [17] asymptotic results for the limiting behavior $\lambda \rightarrow 0$ and $\varepsilon \rightarrow 0$ of the maximal solution branch is presented for these perturbed problems.

Acknowledgements. A. L. was supported by an NSERC (Canada) postgraduate graduate scholarship, and M. J. W. was supported by NSERC (Canada). We are grateful to Prof. J. Wei for several helpful discussions.

\section{REFERENCES}

[1] U. Ascher, R. Christiansen and R. Russell, Collocation Software for Boundary Value ODE's, Math. Comp., 33 (1979), pp. 659-679.

[2] D. Cassani, J. Marcos do Ó And N. Ghoussoub, On a Fourth Order Elliptic Problem with a Singular Nonlinearity, J. Advanced Nonlinear Studies, accepted, (2008).

[3] C. V. Coffman and R. J. Duffin, On the Fundamental Eigenfunctions of a Clamped Punctured Disk, Adv. in Appl. Math., 13:2 (1992), pp. 142-151.

[4] C. V. Coffman and R. J. Duffin, On the Structure of Biharmonic Functions Satisfying the Clamped Plate Conditions on a Right Angle, Adv. in Appl. Math., 1:4 (1980), pp. 373-389.

[5] C. Cowan, P. Esposito and N. Ghoussoub, The Critical Dimension for a Fourth Order Elliptic Problem with Singular Nonlinearity, submitted, (2008).

[6] P. Esposito, N. Ghoussoub and Y. Guo, Compactness Along the Branch of Semi-Stable and Unstable Solutions for an Elliptic Equation with a Singular Nonlinearity, Comm. Pure Appl. Math., 60:12 (2007), pp. 1731-1768.

[7] P. Feng And Z. Zhou, Multiplicity and Symmetry Breaking for Positive Radial Solutions of Semilinear Elliptic Equations Modeling MEMS on Annular Domains, Electron. J. Differential Equations, 146 (2005), 14 pp. (electronic).

[8] N. Ghoussoub and Y. Guo, On the Partial Differential Equations of Electrostatic MEMS Devices: Stationary Case, SIAM J. Math. Anal., 38:5 (2006/07), pp. 1423-1449.

[9] Y. Guo, Z. Pan And M. J. Ward, Touchdown and Pull-In Voltage Behaviour of a MEMS Device with Varying Dielectric Properties, SIAM J. Appl. Math., 66:1 (2005), pp. 309-338.

[10] Z. Guo And J. WeI, Symmetry of Nonnegative Solutions of a Semilinear Elliptic Equation with Singular Nonlinearity, Proc. Roy. Soc. Edinburgh Sect. A, 137:5 (2007), pp. 963-994.

[11] Z. Guo and J. Wei, Infinitely Many Turning Points for an Elliptic Problem with a Singular Nonlinearity, Journ. London. Math. Society, accepted, (2008).

[12] Z. Guo And J. WeI, Asymptotic Behavior of Touchdown Solutions and Global Bifurcations for an Elliptic Problem with a Singular Nonlinearity, Comm. Pure. Appl. Analysis, accepted, (2008).

[13] Z. Guo And J. Wei, Entire Solutions and Global Bifurcations for a Biharmonic Equation with Singular Nonlinearity, accepted, Advances Diff. Equations, (2008).

[14] Z. Guo and J. WeI, On a Fourth Order Nonlinear Elliptic Equation with Negative Exponent, preprint, (2008).

[15] H. B. Keller, Lectures on Numerical Methods in Bifurcation Problems, Tata Institute of Fundamental Research, Bombay, (1987).

[16] F. Lin And Y. Yang, Nonlinear Non-Local Elliptic Equation Modeling Electrostatic Actuation, Proc. Roy. Soc. A, 463 (2007), pp. 1323-1337.

[17] A. Lindsay And M. J. Ward, Asymptotics of Nonlinear Eigenvalue Problems Modeling a MEMS Capacitor: Part II: Multiple Solutions and Singular Asymptotics, to be submitted, European J. Appl. Math., (2008).

[18] J. A. Pelesko and D. H. Bernstein, Modeling MEMS and NEMS, Chapman Hall and CRC Press, (2002)

[19] J. A. Pelesko, Mathematical Modeling of Electrostatic MEMS with Tailored Dielectric Properties, SIAM J. Appl. Math., 62:3 (2002), pp. 888-908. 
[20] J. A. Pelesko, D. Bernstein and J. McCuan, Symmetry and Symmetry Breaking in Electrostatic MEMS, Proceedings of MSM 2003, (2003), pp. 304-307.

[21] J. A. Pelesko and T. A. Driscoll, The Effect of the Small Aspect Ratio Approximation on Canonical Electrostatic MEMS Models, J. Engrg. Math., 53:3-4 (2005), pp. 239-252.

[22] G. SweErs, When is the First Eigenfunction for the Clamped Plate Equation of Fixed Sign?, Electronic J. Differ. Equ. Conf., 6, Southwest Texas State Univ., San Marcos, Texas, (2001), pp. 285-296.

[23] E. Van De Velde and M. J. Ward, Criticality in Reactors Under Domain or External Temperature Perturbation, Proc. R. Soc. Lond. A, 1991:1891 (1991), pp. 341-367

[24] M. J. Ward, W. D. Henshaw and J. Keller, Summing Logarithmic Expansions for Singularly Perturbed Eigenvalue Problems, SIAM J. Appl. Math, 53:3 (1993), pp. 799-828. 
A. E. LINDSAY AND M. J. WARD 